\title{
One-particle irreducibility with initial correlations
}

\author{
Christian Brouder and Frédéric Patras
}

\begin{abstract}
In quantum field theory (QFT), the vacuum expectation of a normal product of creation and annihilation operators is always zero. This simple property paves the way to the classical treatment of perturbative QFT. This is no longer the case in the presence of initial correlations, that is if the vacuum is replaced by a general state. As a consequence, the combinatorics of correlated systems such as the ones occurring in many-body physics is more complex than that of quantum field theory and the general theory has made very slow progress. Similar observations hold in statistical physics or quantum probability for the perturbation series arising from the study of non Gaussian measures. In this work, an analysis of the Hopf algebraic aspects of quantum field theory is used to derive the structure of Green functions in terms of connected and one-particle irreducible Green functions for perturbative QFT in the presence of initial correlations.
\end{abstract}

\section{Introduction}

In quantum field theory (QFT), the initial state is most often the vacuum. Many quantum field concepts, such as Feynman diagrams, the Dyson equation and the Bethe-Salpeter equation rest on the special properties of the vacuum. These desirable concepts extend to special states called quasi-free states [1, 2, 3.

For general initial states, it is not possible to write the Green function in terms of standard Feynman diagrams and the structure of the Green functions is more complex. For example, the Dyson and Bethe-Salpeter equations do not hold. The Dyson equation describes the structure of the two-point interacting Green function. Its extension to non-quasi-free states was discovered by Hall [4] in 1975. However, the equivalent structural equations for $2 n$-point interacting Green functions (with $n>1$ ) is not know for general initial states. The determination of this structure is the main purpose of the present article, with a particular emphasis on the notion of one-particular-irreducibility in this framework.

We stress that the problem of the calculation of Green functions for initial states that are not quasi-free has important applications. For instance, many highly-correlated materials contain transition metals where states of the $3 d$ shell are degenerate. The consequence of this degeneracy is that a small external perturbation can create a very strong change in the state of the system. For instance, a small external magnetic field induces a strong variation in resistance (giant magnetoresistance), that is used to build high-density storage disks. The knowledge of 
the Green functions would enable us to calculate accurately the properties of such materials.

Although we will use mainly the language of QFT, let us mention that the situation where the vacuum is the initial state corresponds in statistical physics to the case of Euclidean measures as showing up e.g. in Euclidean Quantum Field Theory. This is because of the joint use of the Wick theorem (in its simplest, Gaussian, form). For general measures, the problem of determining the fine combinatorial structure of perturbation series was addressed in [5] where, in particular, the combinatorics of truncated (or connected) moment functions was studied intensively. We refer to this article, also for further motivations and examples of computations of thermodynamic limits involving the use of generalized Feynman diagrams as we also use them.

Concretely, in the present article, we investigate the structure of Green functions with Hopf algebraic methods. Hopf algebras have been implicitly used for a long time in statistical physics and quantum field theory: Ruelle [6], Borchers [7] and Stora [8] used a product that is called the convolution product in the Hopf language. Wightman and Challifour [9] defined a triple dot product that was rediscovered only much later in quantum chemistry [10 and was given an algebraic meaning in $\mathbf{1 1}$, where it was called adapted normal product. The work of Wightman and Challifour was summarized by Stora in ref. [8].

The explicit introduction of Hopf algebras by Kreimer and Connes at the level of trees and Feynman diagrams $[\mathbf{1 2}, \mathbf{1 3}$ sparked a reformulation of many quantum field constructions (renormalization [14, 15, 16, Wick's theorem 17], quantization [11, 18], structure of Green functions [19, 20], gauge theory [21]). The result of these efforts is a reasonably complete presentation of QFT in terms of Hopf algebraic concepts 22. Hopf algebras, which are powerful tools to solve combinatorial problems 23, could be expected to help also in the presence of a general state. Indeed, the use of Hopf algebraic methods resulted in the determination of the equation of motion of the Green functions [24] and the description of the relations between general and connected Green functions in the presence of a general state 22 .

The relation between connected and one-particle irreducible (1PI) Green functions, which is the main topic of the present article, is more difficult to understand and depends on the very definition of when a diagram is irreducible. Here, we show that a rather natural definition leads to a complete description of connected Green functions in terms of 1 PI Green functions.

We should point out that our main long-term interest is in the study of the electronic structure of highly correlated materials by means of Green functions, also in the framework of classical (non-relativistic) quantum chemistry. However, we restrict here our study of one-particle-irreducibility to the particular case of local potentials. This covers the potentials of quantum field theories -including QED, which is meaningful for our long-term purposes-, but not the Coulomb potential. There are several ways to remedy this problem and extend the constructions in the present article to time-dependent perturbation theory in many-body physics. For example, as the referee pointed out, our results may be extended to the case of general interactions by means of the notion of block truncation as introduced 
in [5. However, for the sake of simplicity -and since the combinatorics of oneparticle-irreducibility is already intricate enough for local potentials, we decided to postpone the study of electronic systems (and of non-scalar fields) to further work.

The paper starts with a short introduction to Green functions in the presence of initial correlations and to Hopf algebras, followed by the definition of quantum field forms and their convolution logarithm. Then, the relation between forms and connected forms is made explicit, providing the classical relation between general Green functions and connected Green functions. To discuss 1PI functions, we need to generalize a recent work by Mestre and Oeckl 19, 20. Then, a rather natural definition of 1PI functions will be proposed and the Mestre-Oeckl approach will be used to write a connected Green function in terms of these 1PI functions. In the process, universal properties of symmetric functions with respect to Hopf algebra derivations are put forward.

\section{Green functions with initial correlations}

In the present section, we fix the notation and briefly survey the definition of Green functions, emphasizing the role of the initial state.

2.1. Field operators. Quantum fields are operator-valued distributions acting on a Fock space [25]. Here, we describe the construction of Fock space, creation and annihilation operators and the corresponding quantum fields. We start from a self-adjoint operator $h$ acting on a Hilbert space $\mathcal{H}$ and, for notational convenience, we assume that $h$ has a pure point spectrum, so that there is an orthonormal basis $\left|e_{i}\right\rangle$ (with $i \in I$ ) of $\mathcal{H}$ consisting of eigenvectors of $h$. In many applications, the Hilbert space $\mathcal{H}$ is a function space and the eigenvectors (written then preferably with the functional notation $\phi_{n}$ ) are functions of $\mathbf{r}$ (where $\mathbf{r}$ is a point in threedimensional space).

The tensor product of Hilbert spaces is well defined (see Ref. [26] p. 49) and the symmetric Fock space over $\mathcal{H}$ is the Hilbert space $S(\mathcal{H})=\bigoplus_{N=0}^{\infty} S^{N}(\mathcal{H})$, where $S^{N}(\mathcal{H})$ is the $N$-fold symmetric tensor product of $\mathcal{H}$. An orthogonal basis of the vector space $S^{N}(\mathcal{H})$ is provided by the set of vectors

$$
\left|e_{i_{1}}\right\rangle \vee \cdots \vee\left|e_{i_{N}}\right\rangle=\frac{1}{\sqrt{N !}} \sum_{\sigma}\left|e_{i_{\sigma(1)}}\right\rangle \otimes \cdots \otimes\left|e_{i_{\sigma(N)}}\right\rangle
$$

where $\sigma$ runs over the permutations of $N$ elements and where $\left(i_{1}, \ldots, i_{N}\right)$ runs over the subset of $I^{N}$ such that $i_{1} \leq \cdots \leq i_{N}$. In this formula, the symbol $\vee$ denotes the symmetric product and $1 / \sqrt{N}$ ! is a normalization factor.

In many-body theory, $S^{N}(\mathcal{H})$ is called the $N$-particle space of the system and its elements are the $N$-particle states. In particular, $S^{0}(\mathcal{H})$ is a one-dimensional vector space denoted by $\mathbb{C} 1$ in the mathematical literature. In many-body physics and quantum field theory, this unit 1 of the tensor product is denoted by $|0\rangle$, this is the vacuum of the theory (i.e. the state without a particle).

The creation operator $a_{n}^{\dagger}$ is defined as the linear map from $S(\mathcal{H})$ to itself such that, for any basis vector $|u\rangle$ of $S(\mathcal{H}), a_{n}^{\dagger}|u\rangle=c_{n}(u)\left|e_{n}\right\rangle \vee|u\rangle$, where $c_{n}(u)$ is a normalization factor (see for example [27]). It is called a creation operator because it maps $S^{N}(\mathcal{H})$ to $S^{N+1}(\mathcal{H})$ : it adds a new particle to a $N$-particle state. Its adjoint $a_{n}$ is called an annihilation operator. The normalization factor ensures that the commutation relation $a_{m} \circ a_{n}^{\dagger}-a_{n}^{\dagger} \circ a_{m}=\delta_{n m}$ holds, where $\circ$ denotes the composition of operators. 
In functional notation, the corresponding quantum field is the (self-adjoint) operator-valued distribution on the three-dimensional space:

$$
\varphi_{S}(\mathbf{r})=\sum_{n \in I} \phi_{n}(\mathbf{r}) a_{n}+\phi_{n}^{*}(\mathbf{r}) a_{n}^{\dagger} .
$$

This formalism is used to describe scalar particles or photons (up to an additional vector index in that case; recall that a self-adjoint field operator describes a neutral particle, charged scalar or fermion field operators are not self-adjoint). We remind that we focus in the present article on scalar particles (without a charge).

2.2. Adiabatic limit. The adiabatic limit is a very general way of solving the Schrödinger equation for a system described by the Hamiltonian $H=H_{0}+V$ where the eigenstates of $H_{0}$ are known but not those of $H$. The general idea behind the technique is that, for a particle evolving in a potential $V$, the effect of the potential on the motion can be treated (or can be expected to be treated) perturbatively. As we mentioned, besides the perturbative expansions of QFT, the same general idea shows up in the perturbation series of statistical physics [5].

As far as adiabatic limits are concerned, the basic idea is quite simple. We define a time-dependent Hamiltonian $H(t)=H_{0}+\mathrm{e}^{-\epsilon|t|} V$. When $\epsilon$ is small, the interaction $H(t)$ is very slowly switched on from $t=-\infty$ where $H(-\infty)=H_{0}$ to $t=0$ where $H(0)=H$. It is hoped that, if $\epsilon$ is small enough, then an eigenstate of $H_{0}$ is transformed into an eigenstate of $H$.

To implement this picture, the time-dependent Schrödinger equation given by $i \partial\left|\Psi_{S}(t)\right\rangle / \partial t=H(t)\left|\Psi_{S}(t)\right\rangle$ is solved. However, the solution $\left|\Psi_{S}(t)\right\rangle$ is not convenient because it has no limit when $t \rightarrow-\infty$. Therefore, we define $|\Psi(t)\rangle=$ $\mathrm{e}^{i H_{0} t}\left|\Psi_{S}(t)\right\rangle$ that satisfies $i \partial|\Psi(t)\rangle / \partial t=H_{\text {int }}(t)|\Psi(t)\rangle$ with respect to $H_{\text {int }}(t)=$ $\mathrm{e}^{i H_{0} t} V \mathrm{e}^{-i H_{0} t} \mathrm{e}^{-\epsilon|t|}$. Now $H_{\text {int }}(-\infty)=0$ and $|\Psi(-\infty)\rangle$ makes sense. Using $H_{\text {int }}$, we can start from the ground state $\left|\Phi_{0}\right\rangle$ of $H_{0}$ and solve the time-dependent Schrödinger equation with the boundary condition $|\Psi(-\infty)\rangle=\left|\Phi_{0}\right\rangle$. When no eigenvalue crossing takes place, $\left|\Phi_{0}\right\rangle$ should be transformed into the ground state $|\Psi(0)\rangle$ of $H$.

Instead of calculating directly $|\Psi(t)\rangle$ it is convenient to define the unitary operator $U(t)$ as the solution of $i \partial U(t) / \partial t=H_{\text {int }}(t) U(t)$, with the boundary condition $U(-\infty)=1$. Thus, $|\Psi(t)\rangle=U(t)\left|\Phi_{0}\right\rangle$. Note that $U(t)$ depends on $\epsilon$, as $H_{\text {int }}(t)$. But is $\lim _{\epsilon \rightarrow 0} U(0)\left|\Phi_{0}\right\rangle$ an eigenstate of $H$ ? It would if the limit existed, but it does not 30]. However, Gell-Mann and Low [28] discovered in 1951 that

$$
\left|\Psi_{\mathrm{GL}}\right\rangle=\lim _{\epsilon \rightarrow 0} \frac{U(0)\left|\Phi_{0}\right\rangle}{\left\langle\Phi_{0}|U(0)| \Phi_{0}\right\rangle}
$$

exists and is an eigenstate of $H$. A mathematical proof of this fact for reasonable Hamiltonians came much later [29]. Notice that the above scheme works when the ground state of $H_{0}$ is non degenerate. When it is degenerate, the problem is more subtle [33, 34] and the limit $\epsilon \rightarrow 0$ only exists when $\left|\Phi_{0}\right\rangle$ is properly chosen 31, 32 .

2.3. Green functions. We now come to the heart of QFT: the calculation of Green functions (or moment functions). Green functions are important because they allow for the calculation of practically all relevant physical observables: energy, charge density, transport coefficients, current density, dielectric constants, etc. In particular, they show up naturally in the perturbative expansions arising from 
adiabatic limits. If we could calculate Green functions exactly, we would know all interesting properties of matter. Of course, as far as many-body theory is concerned, we cannot calculate exact Green functions for realistic materials, but non-perturbative approximations are now used with great success [35].

When the dynamics of the particles is described by a one-body Hamiltonian $H_{0}$, the $n$-point Green function for scalar particles is defined by

$$
G_{n}^{0}\left(x_{1}, \ldots, x_{n}\right)=\left\langle\Phi_{0}\left|T\left(\varphi\left(x_{1}\right) \ldots \varphi\left(x_{n}\right)\right)\right| \Phi_{0}\right\rangle,
$$

where $x=(t, \mathbf{r}), T$ is the time-ordering operator and $\varphi(x)$ is related to $\varphi_{S}(\mathbf{r})$ by

$$
\varphi(x)=\mathrm{e}^{i H_{0} t} \varphi_{S}(\mathbf{r}) \mathrm{e}^{-i H_{0} t}=\sum_{n \in I} \mathrm{e}^{-i \epsilon_{n} t} \phi_{n}(\mathbf{r}) a_{n}+\mathrm{e}^{i \epsilon_{n} t} \phi_{n}^{*}(\mathbf{r}) a_{n}^{\dagger},
$$

where the $\phi_{n}(\mathbf{r})$ are eigenvectors of $H_{0}$ with associated eigenvalues $\epsilon_{n}$. The timeordering operator orders the quantum fields $\varphi\left(x_{1}\right), \ldots, \varphi\left(x_{n}\right)$ so that the field $\varphi\left(x_{i}\right)$ is on the left of $\varphi\left(x_{j}\right)$ if $t_{i}$ is greater (i.e. later) than $t_{j}$. For example $T\left(\varphi\left(x_{1}\right) \varphi\left(x_{2}\right)\right)=\varphi\left(x_{1}\right) \varphi\left(x_{2}\right)$ if $t_{1}>t_{2}$ and $T\left(\varphi\left(x_{1}\right) \varphi\left(x_{2}\right)\right)=\varphi\left(x_{2}\right) \varphi\left(x_{1}\right)$ if $t_{1}<t_{2}$.

When the dynamics of the particles is described by a Hamiltonian $H=H_{0}+V$, where $H_{0}$ is one-body, the expression for the Green function becomes [36, 27]

$$
G_{n}\left(x_{1}, \ldots, x_{n}\right)=\frac{\left\langle\Phi_{0}\left|T\left(\varphi\left(x_{1}\right) \ldots \varphi\left(x_{n}\right) \mathrm{e}^{-i \int H_{\text {int }}(t) \mathrm{d} t}\right)\right| \Phi_{0}\right\rangle}{\left\langle\Phi_{0}\left|T\left(\mathrm{e}^{-i \int H_{\text {int }}(t) \mathrm{d} t}\right)\right| \Phi_{0}\right\rangle},
$$

where $H_{\text {int }}(t)=\mathrm{e}^{i H_{0} t} V \mathrm{e}^{-i H_{0} t} \mathrm{e}^{-\epsilon|t|}$ and the limit $\epsilon \rightarrow 0$ is implicitly taken. This generalizes to non-scalar particles and, in the example of the non-relativistic electrons,

$$
H_{\text {int }}(t)=\mathrm{e}^{-\epsilon|t|} \frac{1}{2} \int \mathrm{d} \mathbf{r} \mathrm{d} \mathbf{r}^{\prime} \psi^{\dagger}(t, \mathbf{r}) \psi^{\dagger}\left(t, \mathbf{r}^{\prime}\right) V_{e e}\left(\mathbf{r}-\mathbf{r}^{\prime}\right) \psi\left(t, \mathbf{r}^{\prime}\right) \psi(t, \mathbf{r}) .
$$

We are now ready to enter correlated systems. Assuming that the initial state $\left|\Phi_{0}\right\rangle$ is the free field vacuum $|0\rangle$ implies the classical expansion of Green functions in terms of Feynman propagators and, ultimately, of (usual) Feynman diagrams. In many-body theory, the use of this decomposition of Green function into sums of (usual) Feynman diagrams is restricted to very specific states $\left|\Phi_{0}\right\rangle$ called quasi-free states. For the other states (or, equivalently, for the study of general functional measures in statistical physics), the structure of Green functions is more complex. Let us give a simple example. We can define the quantity $D_{4}\left(x_{1}, x_{2}, x_{3}, x_{4}\right)$ by

$$
\begin{aligned}
G_{4}^{0}\left(x_{1}, x_{2}, x_{3}, x_{4}\right)= & G_{2}^{0}\left(x_{1}, x_{2}\right) G_{2}^{0}\left(x_{3}, x_{4}\right)+G_{2}^{0}\left(x_{1}, x_{3}\right) G_{2}^{0}\left(x_{2}, x_{4}\right) \\
& +G_{2}^{0}\left(x_{1}, x_{4}\right) G_{2}^{0}\left(x_{2}, x_{3}\right)+D_{4}\left(x_{1}, x_{2}, x_{3}, x_{4}\right) .
\end{aligned}
$$

When the initial state is the vacuum or a quasi-free state, the term $D_{4}$ is zero. For a general initial state, it is not.

For a fermionic system, a term $D_{4}$ can be defined similarly. It is absent when the ground state of $H_{0}$ can be written as a Slater determinant. It is present when the ground state of $H_{0}$ is degenerate, as in open shell systems. In that case, $\left|\Phi_{0}\right\rangle$ can be written as a linear combination of Slater determinants and $D_{4}$ describes the correlation between these determinants. The presence of several Slater determinants in the initial state is rather catastrophic for many-body theory. Yaris and

\footnotetext{
${ }^{1}$ For non-equilibrium systems, additional complications come from the fact that time-ordered products must be defined over a closed time path [37. However, this does not change the combinatorial aspects of the problem.
} 
Taylor summarized the situation [38]: "The inability to handle open-shell systems is a ubiquitous problem in many-body theory. It basically arises when one cannot find a single-determinant unperturbed ground state which connects to the exact ground state when the residual interaction is adiabatically switched on. When this situation holds, one cannot properly define occupied and unoccupied single-particle states, Wick's theorem does not hold, and Dyson equations, Bethe-Salpeter equations, etc. do not exist." To this list one can add that ordinary Feynman diagrams and the Gell-Mann and Low formula are lost. In other words, most of the tools of quantum field theory break down. Since the seminal work by Bloch and Horowitz in 1958 [39], many works were devoted to the rebuilding of these tools. Morita discovered a modified version of the Gell-Mann and Low theorem 40, Fujita defined generalized Feynman diagrams [41], Hall derived a Dyson equation for degenerate systems [4]. Since then, progress has been quite slow because of the combinatorial complexity of the problem.

To illustrate this complexity, we first describe the generalized Feynman diagrams introduced by Fujita [41], Hall [4] and Djah et al. [5]. For bosonic and fermionic systems, $D_{4}$ can be thought of as a sort of 4-point Feynman propagator, as $D_{2}(x, y)=G_{2}^{0}(x, y)$ is the 2-point Feynman propagator. We shall see that $D_{4}$ plays the role of a cumulant, as in the decomposition of a distribution function. Higher order Green functions $G_{2 n}^{0}$ give rise to higher order propagators $D_{2 n}$ and the precise relation between them will be described in the following. In standard quantum field theory, the Green function of the interacting system can be written by adding all possible Feynman diagrams involving the two-point propagator $D_{2}$. When the initial state is not quasi-free, the Green function is written as the sum of all possible Feynman diagrams involving 2-point, 4-point, and $2 n$-point propagators for arbitrary $n$. An example will be given in figure 2 of this paper.

\section{Hopf algebra}

We do not provide the general definition of a Hopf algebra (see e.g. [42]) and consider only the special case of the symmetric Hopf algebra $S(V)=\bigoplus_{n} S^{n}(V)=$ $\bigoplus_{n} V^{\otimes n} / \mathcal{S}_{n}$, where $V$ is a complex vector space and where $\mathcal{S}_{n}$, the symmetric group of order $n$, acts by permutation on the components of the tensor power $V^{\otimes n}$. The commutative product of $S(V)$ is denoted by concatenation. The counit is the linear map $\varepsilon: S(V) \rightarrow \mathbb{C}$ defined by $\varepsilon(1)=1, \varepsilon(u)=0$ if $u \in S^{n}(V)$ with $n>0$. The coproduct is the linear map $\Delta: S(V) \rightarrow S(V) \otimes S(V)$ determined by $\Delta 1=1 \otimes 1$, $\Delta a=1 \otimes a+a \otimes 1$ for $a \in V$ and $\Delta(u v)=(\Delta u)(\Delta v)$, for $u$ and $v$ in $S(V)$. We employ the strengthened Sweedler notation for the coproduct [43]: $\Delta u=u_{(1)} \otimes u_{(2)}$. Recall that there is an implicit summation in the notation, which does not lead to ambiguities when handled correctly: the right hand side should be understood not as the mere tensor product of two elements in $S(V)$ but as a sum of such elements (so that e.g. $a_{(1)} \otimes a_{(2)}=1 \otimes a+a \otimes 1$ for $a \in V$ ). More generally, an expression such as $u_{(1)} v_{(1)} \otimes u_{(2)} v_{(2)}$, which stands for $\Delta(u) \Delta(v)=\Delta(u v)$, contains an implicit double summation and should be understood as: $\left(u_{(1)} \otimes u_{(2)}\right)\left(v_{(1)} \otimes v_{(2)}\right)$, and similarly for expressions of higher orders.

The iterated coproducts $\Delta^{k}$ are defined by $\Delta^{0}=\mathrm{id}, \Delta^{1}=\Delta$ and $\Delta^{k+1}=$ $\left(\mathrm{id}^{\otimes k} \otimes \Delta\right) \Delta^{k}$. Their action on an element $u$ of $S(V)$ is denoted by $\Delta^{k} u=u_{(1)} \otimes$ $\cdots \otimes u_{(k+1)}$. For any $u \in S(V)$, the reduced coproduct is the map $\triangleq: S(V) \rightarrow$ 
$S(V) \otimes S(V)$ such that $\underline{\Delta} u=\Delta u-1 \otimes u-u \otimes 1$. The iterated reduced coproducts $\underline{\Delta}^{k}$ are defined by $\underline{\Delta}^{0}=\mathrm{id}, \underline{\Delta}^{1}=\underline{\Delta}$ and $\underline{\Delta}^{k+1}=\left(\mathrm{id}{ }^{\otimes k} \otimes \underline{\Delta}\right) \underline{\Delta}^{k}$. Their action on an element $u$ of $S(V)$ is denoted by $\underline{\Delta}^{k} u=u_{(\underline{1})} \otimes \cdots \otimes u_{(k+1)}$. The coproduct and the reduced coproduct are cocommutative, that is:

$$
\Delta(u)=u_{(1)} \otimes u_{(2)}=u_{(2)} \otimes u_{(1)}, \quad \underline{\Delta}(u)=u_{(\underline{1})} \otimes u_{(\underline{2})}=u_{(\underline{2})} \otimes u_{(\underline{1})} .
$$

The coproduct is an algebra morphism, but the reduced coproduct is not. Its relation with the product is described by the following simple and useful lemma.

Lemma 3.1. If $a \in V$ and $u \in S(V)$, then

$$
\underline{\Delta}(a u)=a \otimes u+u \otimes a+a u_{(\underline{1})} \otimes u_{(\underline{2})}+u_{(\underline{1})} \otimes a u_{(\underline{2})},
$$

and, for $k>1$,

$$
\underline{\Delta}^{k}(a u)=a \otimes \underline{\Delta}^{k-1} u+a u_{(\underline{1})} \otimes \underline{\Delta}^{k-1} u_{(\underline{2})}+u_{(\underline{1})} \otimes \underline{\Delta}^{k-1}\left(a u_{(\underline{2})}\right) .
$$

More explicitly, for $k>0$,

$$
\begin{aligned}
\underline{\Delta}^{k}(a u)= & \sum_{i=1}^{k+1} u_{(\underline{\underline{1}})} \otimes \cdots \otimes u_{(\underline{\underline{i-1})}} \otimes a \otimes u_{(\underline{\underline{i}})} \otimes \cdots \otimes u_{(\underline{k})} \\
& +\sum_{i=1}^{k+1} u_{(\underline{\underline{1}})} \otimes \cdots \otimes u_{\underline{(\underline{i-1})}} \otimes a u_{(\underline{\underline{i}})} \otimes u_{(\underline{i+1)}} \otimes \cdots \otimes u_{(\underline{k+1)}},
\end{aligned}
$$

where the terms $i=1$ and $i=k+1$ are $a \otimes \Delta^{k} u$ and $\underline{\Delta}^{k} u \otimes a$ in the first sum and $\left(a \otimes 1^{\otimes k}\right) \underline{\Delta}^{k} u$ and $\left(1^{\otimes k} \otimes a\right) \underline{\Delta}^{k} u$ in the second term.

For an arbitrary $u \in S^{n}(V), n>0$ and $v \in S(V)$, we also have:

$$
\begin{aligned}
\Delta^{k}(u v) & =u_{(1)} v_{(1)} \otimes \cdots \otimes u_{(k)} v_{(k)} \\
& =\sum_{1 \leq p \leq k} \sum_{1 \leq i_{1}<\cdots<i_{p} \leq k} v_{(1)} \otimes \cdots \otimes u_{(\underline{1})} v_{\left(i_{1}\right)} \otimes \cdots \otimes u_{(\underline{p})} v_{\left(i_{p}\right)} \otimes \cdots \otimes v_{(k)} .
\end{aligned}
$$

\section{Green functions for quasi-free states}

Let $V$ be the vector space generated by the symbols $\varphi(x)$, where $x$ runs over points of $\mathbb{R}^{d}$. In physical terms, $\varphi$ should be thought of as a free bosonic field operator, that is, as an operator-valued distribution (think of the quantum fields $\phi_{\mathbf{S}}(\mathbf{r})$ ). Our forthcoming developments can be adapted easily to fermionic systems, the adaptation amounting mathematically to replacing the symmetric algebra $S(V)$ by the exterior (or Grassmann) algebra $\Lambda(V)$, see [1].

Defining a time-ordered product of fields at the same point gives rise to major difficulties and is the subject of renormalization 44. Here, we take advantage of the fact that the combinatorics of Green functions is in many respects a self-contained topic and leave aside these questions (renormalization, operator product expansion). We will therefore treat powers of fields such as $\varphi^{4}(x)$ as formal expressions, that is as monomials belonging to the symmetric Hopf algebra $S(V) \supset S^{4}(V)$. Note that $\varphi^{0}(x)=1$ is the unit of the algebra $S(V)$.

\footnotetext{
${ }^{2}$ In another paper 22$]$, an algebra different from $S(V)$ was used, where $\varphi^{0}(x)$ was not the unit of the algebra, in order to obtain some desirable coalgebraic properties. That alternative construction considers the field products $\varphi^{n}(x)$ as the basis of a Hopf algebraic fiber at $x$. However such a point of view is not required in the present paper.
} 
4.1. Convolution. In this section we survey some Hopf algebraic concepts that provide a startling simplification of the decomposition of the expectation value of time-ordered products in terms of Feynman diagrams. We define a form as a linear map from $S(V)$ to $\mathbb{C}$. A unital form is a form $\rho$ such that $\rho(1)=1$. In our context, that is when $\varphi(x)$ is the quantum field of QFT or many-body theory, unital forms are defined from states of $H_{0}$ : if $|\Phi\rangle$ is a normalized state and $u \in S(V)$, then $\rho(u)=\langle\Phi|T(u)| \Phi\rangle$ is a unital form because it is obviously linear and $\rho(1)=\langle\Phi|1| \Phi\rangle=1$. The unital form corresponding to the vacuum is denoted by $\rho_{0}$, so that $\rho_{0}(u)=\langle 0|T(u)| 0\rangle$.

To express $\rho_{0}(u)$ in Hopf algebraic terms, we first need a few definitions. The convolution product of two forms $\rho$ and $\sigma$ is the form $\rho * \sigma$ defined by $(\rho * \sigma)(u)=$ $\rho\left(u_{(1)}\right) \sigma\left(u_{(2)}\right)$. Notice that, because of the commutativity and cocommutativity of $S(V), \sigma * \rho=\rho * \sigma$. The space of unital forms equipped with the convolution product is a commutative group, denoted by $\mathcal{S}$, whose unit is the counit $\varepsilon$.

The $n$-th convolution power of a form $\rho$ is the form $\rho^{* n}$ defined recursively by $\rho^{* 0}=\varepsilon, \rho^{* 1}=\rho$ and $\rho^{*(n+1)}=\rho^{* n} * \rho$. The convolution exponential of a form $\rho$ is the form $\mathrm{e}^{* \rho}$ defined by

$$
\mathrm{e}^{* \rho}=\sum_{n=0}^{\infty} \frac{\rho^{* n}}{n !} .
$$

The convolution logarithm $\log ^{*} \rho$ of the form $\rho$ is the form defined by

$$
\log ^{*} \rho=\sum_{n=1}^{\infty} \frac{(-1)^{n+1}}{n}(\rho-\epsilon)^{* n} .
$$

Note that, if $\rho$ is a unital form, $\log ^{*} \rho$ satisfies $\log ^{*} \rho(1)=0$. A form $\sigma$ such that $\sigma(1)=0$ is called an infinitesimal form because it is the logarithm of a unital form. In the present paper, the convolution exponential is always applied to infinitesimal forms. Note that, if $\sigma=\log ^{*} \rho$, then $\mathrm{e}^{* \sigma}=\rho$. In other words, convolution exponential and convolution logarithm are inverse functions of each other. At last, note that, if $\alpha$ and $\beta$ are two unital forms with convolution logarithms $a$ and $b$, then $\alpha * \beta=\mathrm{e}^{* a} * \mathrm{e}^{* b}=\mathrm{e}^{*(a+b)}$.

4.2. Expansion in Feynman diagrams. In standard quantum field theory, Wick's theorem states that, if $u=\varphi^{k_{1}}\left(x_{1}\right) \ldots \varphi^{k_{n}}\left(x_{n}\right),<0|T(u)| 0>$ is calculated as the sum of all pairings of $k_{1}$ times the point $x_{1}, \ldots, k_{n}$ times the point $x_{n}$. A pairing is the choice of a pair of different points represented graphically as a line and analytically as a Feynman propagator. Graphically, $\rho_{0}(u)=\langle 0|T(u)| 0\rangle$ is therefore represented by the sum of all the graphs with $n$ vertices labeled by $x_{1}, \ldots, x_{n}$ such that $k_{i}$ edges are incident to the vertex labeled by $x_{i}$, for $i=1, \ldots, n$. Each graph is weighted by a proper combinatorial factor.

To express $\rho_{0}(u)$ in Hopf algebraic terms, we define the infinitesimal form $\tau$ by

$$
\tau\left(\varphi\left(x_{1}\right) \varphi\left(x_{2}\right)\right):=D_{F}\left(x_{2}-x_{1}\right) \text { if } x_{1} \neq x_{2},
$$

and

$$
\tau\left(\varphi\left(x_{1}\right) \ldots \varphi\left(x_{n}\right)\right):=0 \text {, if } n \neq 2 \text { or } n=2 \text { and } x_{1}=x_{2}
$$


The form $\tau$ is called the Feynman form. The function $D_{F}$ is defined by

$$
D_{F}(x)=\int \frac{d^{4} p}{(2 \pi)^{4}} \frac{i}{p^{2}-m^{2}+i \varepsilon} e^{-i(p \cdot x)} .
$$

We can now restate Wick's theorem algebraically:

Theorem 4.1. (6, 6, 11, 22]) The unital form $\rho_{0}$ is the convolution exponential of the Feynman form :

$$
\rho_{0}=e^{* \tau}
$$

This theorem extends to the case of quasi-free states 45, the only change is that $\tau$ is now defined by

$$
\tau\left(\varphi\left(x_{1}\right) \varphi\left(x_{2}\right)\right):=D_{2}\left(x_{1}, x_{2}\right) \text { if } x_{1} \neq x_{2},
$$

and

where $D_{2}\left(x_{1}, x_{2}\right)=\left\langle\Phi\left|T\left(\varphi\left(x_{1}\right) \varphi\left(x_{2}\right)\right)\right| \Phi\right\rangle$.

$$
\tau\left(\varphi\left(x_{1}\right) \ldots \varphi\left(x_{n}\right)\right):=0 \text {, if } n \neq 2 \text { or } n=2 \text { and } x_{1}=x_{2},
$$

\section{Green functions for general states}

Most often the relevant object to deal with in perturbative expansions is actually not the unital form $\rho$ built from the ground state $|\Phi\rangle$ (or, abstractly, the group $\mathcal{S}$ ) but its convolution logarithm $r$ (resp. the corresponding commutative Lie algebra $\mathcal{L}$ ). The infinitesimal form $r$ is called the cumulant form. As we shall see in section 6.2, this is exactly what we need to calculate the Green functions in the presence of initial correlations.

The theorem (4.1) of the previous section trivially generalizes to arbitrary states and unital forms:

Theorem 5.1. (6, 7, 5) The unital form $\rho$ is the convolution exponential of its cumulant form :

$$
\rho=e^{* r} \text {. }
$$

Although our approach is not the usual one, writing $\rho$ as $\mathrm{e}^{* r}$ is in fact quite common in physics. The notion of a cumulant form is related to the cumulant expansion, and expresses the generalized Wick theorem used for solving the Anderson model 46. Moreover, it is a way to isolate the singularities of the forms because a natural property of a quantum field is that $r\left(\varphi\left(x_{1}\right) \ldots \varphi\left(x_{n}\right)\right)$ is a smooth function of $x_{1}, \ldots, x_{n}$, except possibly for $n=2$ (see 447, as well as 448 for a related result in many-body theory).

Finally, an observation that will prove essential in our forthcoming developments: all our previous reasonings suggest that a unital form $\rho=\mathrm{e}^{* r}$ should be dealt with by means of generalized propagators in the same way as vacuum expectations of time-ordered products of free fields are dealt with by means of 2-point Green functions and Feynman propagators in the usual picture of QFT. However, whereas the Feynman propagator, which is associated to the unique non trivial component of $\tau$ (recall that $\tau=0$ on $S^{i}(V)$ for any $i \neq 2$ ), is described graphically by a line linking two vertices, we may have now $r\left(\varphi\left(x_{1}\right) \ldots \varphi\left(x_{n}\right)\right) \neq 0$ with $n \neq 2$. Accordingly, we shall represent graphically the " $n$-point propagator"

\footnotetext{
${ }^{3}$ When $x_{1}$ and $x_{2}$ are separated by a light-like interval, the definition of $\tau$ does not make sense and $D_{F}$ should be replaced by a smooth regularization. We do not enter into these details here since we consider only the combinatorial aspects of the problem.
} 
$D_{n}\left(x_{1}, \ldots, x_{n}\right)=r\left(\varphi\left(x_{1}\right) \ldots \varphi\left(x_{n}\right)\right)^{4}$ by a white dot with $n$ edges linked to the $n$ vertices $x_{1}, \ldots, x_{n}$, as shown in figure 1 (a similar convention was used by Djah et al. [5]).

$$
D_{3}(x, y, z)=\underbrace{z}_{x}
$$

Figure 1. The generalized propagator $D_{3}(x, y, z)=$ $r(\varphi(x) \varphi(y) \varphi(z))$

As we already mentioned, we can also consider $\rho=\mathrm{e}^{* r}$ as a generalization of Wick's theorem when the latter is stated algebraically. The same observation holds for graphical statements of the theorem: we saw that, in standard quantum field theory, Wick's theorem states that, if $u=\varphi^{k_{1}}\left(x_{1}\right) \ldots \varphi^{k_{n}}\left(x_{n}\right),\langle 0|T(u)| 0\rangle$ is calculated as the sum of all pairings of $k_{1}$ times the point $x_{1}, \ldots, k_{n}$ times the point $x_{n}$. In the many-body context $\mathrm{e}^{* \tau}(u)$ is replaced by $\mathrm{e}^{* r}(u)$. This amounts to say that we write $\mathrm{e}^{* r}(u)$ as the sum of all ways to partition the multiset made of $k_{1}$ times point $x_{1}, \ldots, k_{n}$ times point $x_{n}$ into sub-multisets of any multiplicity (i.e. not only pairs and not only different points). See figure 2 for an example. The $n$-point propagators are then a convenient way to represent these sub-multisets.

To conclude this section, we state three easy but important lemmas

Lemma 5.1. If $a \in V$ and $E=\mathrm{e}^{a}$, then for any form $\rho$ with logarithm $r$, we have $\rho(E)=\mathrm{e}^{r(E)}$.

Lemma 5.2. If $a \in V$ and $u \in S(V)$, then, for any linear map $r: S(V) \rightarrow \mathbb{C}$ such that $r(1)=0$,

$$
\mathrm{e}^{* r}(a u)=\sum r\left(a u_{(1)}\right) \mathrm{e}^{* r}\left(u_{(2)}\right)
$$

More generally,

Lemma 5.3. For any $u \in \operatorname{ker} \epsilon$ and any $v$ in $S(V)$,

$$
\mathrm{e}^{* r}(u v)=\sum_{k=1}^{\infty} \frac{1}{k !} r\left(u_{(\underline{1})} v_{(1)}\right) \ldots r\left(u_{(\underline{k})} v_{(k)}\right) \mathrm{e}^{* r}\left(v_{(k+1)}\right) .
$$

Proof. The first lemma is a simple consequence of the fact that $E$ is group-like (that is, $\Delta(E)=E \otimes E$ ). The second lemma was shown in [22], it follows from the cocommutativity of the coproduct and the fact that $a$ is a primitive element (that is, $\Delta(a)=1 \otimes a+a \otimes 1)$. The third lemma follows from the last identity in Lemma 3.1. from the properties of the binomial coefficients, and from the cocommutativity of the coproduct.

The first lemma is often used with $a=\int j(x) \varphi(x) \mathrm{d} x$ (up to a suitable extension of the definition of $V$ when the function $j(x)$ has not a discrete support). In that case, it relates the generating function of the moments of $\rho$ to that of the moments

\footnotetext{
${ }^{4}$ The definition of $D_{n}$ generalizes the definition of $D_{4}$ in section 2.3 -this should be clear from our forthcoming developments.
} 
of $r$. The second and third lemmas provide powerful tools for the recursive proof of the properties of $\mathrm{e}^{* r}$. Notice in particular that, using the last Lemma with $v=1$ :

$$
\rho\left(a^{n}\right)=\sum_{k=1}^{n} \frac{1}{k !} \sum_{i_{1}+\cdots+i_{k}=n} \frac{n !}{i_{1} ! \ldots i_{k} !} r\left(a^{i_{1}}\right) \ldots r\left(a^{i_{k}}\right),
$$

where, for $p=1, \ldots, k, i_{p}>0$. A formula with less terms can be given using the Faà di Bruno coefficients:

$$
\rho\left(a^{n}\right)=\sum_{\alpha} \frac{n ! r\left(a^{1}\right)^{\alpha_{1}} \ldots r\left(a^{n}\right)^{\alpha_{n}}}{\alpha_{1} !(1 !)^{\alpha_{1}} \alpha_{2} !(2 !)^{\alpha_{2}} \ldots \alpha_{n} !(n !)^{\alpha_{n}}},
$$

where $\left(\alpha_{1}, \ldots, \alpha_{n}\right)$ are nonnegative integers such that $\sum_{i} i \alpha_{i}=n$. For the partition represented by $\alpha, n$ is cut into $k=\sum_{i} \alpha_{i}$ parts. For example, $\rho(a)=r(a), \rho\left(a^{2}\right)=$ $r\left(a^{2}\right)+r(a)^{2}, \rho\left(a^{3}\right)=r\left(a^{3}\right)+3 r(a) r\left(a^{2}\right)+r(a)^{3}$.

\section{Connected forms}

In quantum field theory, an important simplification comes from the fact that all physical quantities can be expressed in terms of connected diagrams. We first define the notion of a connected form by analogy with that of a connected diagram. A monomial of $S(V)$ can always be written $u=\varphi^{n_{1}}\left(x_{1}\right) \ldots \varphi^{n_{k}}\left(x_{k}\right)$, where all points $x_{i}$ are distinct. Now, for any form $\rho$ with convolution logarithm $r$, we use eq. (4) to expand $\rho(u)$ in terms of $r$.

Proposition 6.1. We have

$$
\begin{aligned}
\rho(u)=\mathrm{e}^{* r}(u)= & \sum_{l \in \mathbb{N}} \frac{1}{l !} \sum_{\substack{n_{i}^{1}+\ldots+n_{i}^{l}=n_{i} \\
i=1 \ldots k}} \prod_{i=1}^{k} \frac{n_{i} !}{n_{i}^{1} ! \ldots n_{i}^{l} !} \\
& \times r\left(\varphi^{n_{1}^{1}}\left(x_{1}\right) \ldots \varphi^{n_{k}^{1}}\left(x_{k}\right)\right) \ldots r\left(\varphi^{n_{1}^{l}}\left(x_{1}\right) \ldots \varphi^{n_{k}^{l}}\left(x_{k}\right)\right),
\end{aligned}
$$

where, for $i=1, \ldots, k$, the sum is over all the l-tuples of nonnegative integers $\left(n_{i}^{1}, \ldots, n_{i}^{l}\right)$ such that $n_{i}^{1}+\cdots+n_{i}^{l}=n_{i}$.

Although a straightforward application of the Hopf algebra formalism, the result is important since it allows us to compute the multiplicity of a graph -or symmetry factor- in the Feynman diagrammatic perturbative expansion of amplitudes. We refer for example to the expansion of the connected Green functions for $\varphi^{3}$ theory with an arbitrary ground state in the present section of the article.

Let us consider a term $t:=r\left(\varphi^{n_{1}^{1}}\left(x_{1}\right) \ldots \varphi^{n_{k}^{1}}\left(x_{k}\right)\right) \ldots r\left(\varphi^{n_{1}^{l}}\left(x_{1}\right) \ldots \varphi^{n_{k}^{l}}\left(x_{k}\right)\right)$ of $\mathrm{e}^{* r}(u)$. We say that $x_{i} \cong_{t} x_{j}, 1 \leq i, j \leq k$ if there exists $m \leq l$ with $n_{i}^{m} n_{j}^{m} \neq 0$. The transitive closure $\equiv_{t}$ of the binary relation $\cong_{t}$ defines the connectedness of $t: t$ is said to have $n$ connected components if there are $n$ equivalence classes associated to the equivalence relation $\equiv_{t}$. The connected component of $x_{i}$ in $t$ is defined similarly as the product of all the $r\left(\varphi^{n_{1}^{m}}\left(x_{1}\right) \ldots \varphi^{n_{k}^{m}}\left(x_{k}\right)\right)$ with $n_{j}^{m} \neq 0$ for at least one coefficient $j$ with $x_{i} \equiv_{t} x_{j}$. When $n=1$ (resp. $n \neq 1$ ), we also say that the term $t$ is connected (resp. disconnected). Let us take a simple example. For $u=\varphi(x) \varphi^{2}(y)$, we have $\rho(u)=r(\varphi(x)) r\left(\varphi^{2}(y)\right)+r(\varphi(x)) r(\varphi(y))^{2}+2 r(\varphi(x) \varphi(y)) r(\varphi(y))+r\left(\varphi(x) \varphi^{2}(y)\right)$, where the first two terms are disconnected (they actually have two connected components). The connected components of $y$ in the four terms are respectively 
$r\left(\varphi^{2}(y)\right), r(\varphi(y))^{2}, r(\varphi(x) \varphi(y)) r(\varphi(y))$ and $r\left(\varphi(x) \varphi^{2}(y)\right)$. The definition of connected form is actually best formulated in algebraic terms: this is the purpose of the next section.

6.1. Another coproduct on $S(V)$. As we have just seen, a pedestrian definition of connectedness makes an essential use of the fact that some points $x_{i}$ are equal or distinct. In order to reflect this distinction, we define a new coproduct, the disconnecting coproduct $\delta: S(V) \rightarrow S(V) \otimes S(V)$.

So we write a monomial of $S(V)$ as $u=\varphi^{n_{1}}\left(x_{1}\right) \ldots \varphi^{n_{k}}\left(x_{k}\right)$, where all points $x_{i}$ are distinct, and we define the coproduct of $u$ as follows: $\delta \varphi^{n}(x)=1 \otimes \varphi^{n}(x)+$ $\varphi^{n}(x) \otimes 1$ if $k=1$, and $\delta u=\delta\left(\varphi^{n_{1}}\left(x_{1}\right)\right) \delta\left(\varphi^{n_{2}}\left(x_{2}\right)\right) \ldots \delta\left(\varphi^{n_{k}}\left(x_{k}\right)\right)$ if $k>1$. Notice that this coproduct is coassociative and cocommutative but is not an algebra morphism, because $\delta\left(\varphi^{2}(x)\right) \neq(\delta(\varphi(x)))^{2}$. Since $\delta$ is coassociative and cocommutative, we may still define an associative, commutative and unital product $\hat{*}$, the disconnecting convolution product, on $\operatorname{Lin}(S(V), \mathbb{C})$ :

$$
\forall(f, g) \in \operatorname{Lin}(S(V), \mathbb{C}), f \hat{*} g:=\pi \circ(f \otimes g) \circ \delta,
$$

where $\pi$ denotes the product: $\pi \circ(f \otimes g)(u \otimes v)=f(u) g(v)$. The unit of $\hat{*}$ is the same as the unit of $*$ (the projection map $\varepsilon$ from $S(V)$ to $\mathbb{C} \subset S(V)$ ). To distinguish between the two products $*$ and $\hat{*}$, we write the operations involving $\hat{*}$ with a superscript $\hat{*}$ : for example, we write $\log ^{\hat{*}}$, and so on.

The relation between $\delta$ and $\Delta$ is investigated in 22]. The reduced coproduct $\underline{\delta}$ and the iterated coproduct $\delta^{k}$ are defined as in section 3. The enhanced Sweedler notation for the disconnecting coproduct is $\delta u=u_{\{1\}} \otimes u_{\{2\}}$ and $\underline{\delta} u=u_{\{\underline{1}\}} \otimes u_{\{\underline{2}\}}$.

The new coproduct $\delta$ enables us to give an algebraic definition of the connected form $\rho_{c}$ corresponding to the unital form $\rho$ :

$$
\rho_{c}=\log ^{\hat{*}}(\rho)
$$

that is,

$$
\forall u \in S(V), \rho_{c}(u)=\sum_{n=1}^{\infty} \frac{(-1)^{n+1}}{n}(\rho-\varepsilon)\left(u_{\{1\}}\right) \ldots(\rho-\varepsilon)\left(u_{\{n\}}\right) .
$$

This identity can be understood as a linked cluster theorem for forms. The same problem was addressed in [5, Sect.6] with another approach: in that article, the authors introduce two notions of truncated moment functions, indicated respectively by an exponent $T$ and $(T)$. This corresponds roughly, at the Hopf algebraic level, to our distinction between the two coproducts $\Delta$ and $\delta$. We thank the referee for pointing out to us this point and refer to [5] for further insights on truncated moment functions.

Pedantically, the set of connected forms is defined as the image of the group of unital forms under the map $\log ^{\hat{*}}$. The two sets are in bijective correspondence and, reciprocally, we can express any unital form $\rho$ in terms of $\rho_{c}$ by

$$
\rho=\mathrm{e}^{\hat{*} \rho_{c}}
$$

or:

$$
\forall u \in S(V), \rho(u)=\varepsilon(u)+\sum_{n=1}^{\infty} \frac{1}{n !} \rho_{c}\left(u_{\{1\}}\right) \ldots \rho_{c}\left(u_{\{n\}}\right) .
$$

For example, $\rho_{c}\left(\varphi^{n}(x)\right)=\rho\left(\varphi^{n}(x)\right)$ for $n>0$ and

$$
\rho_{c}\left(\varphi^{m}(x) \varphi^{n}(y)\right)=\rho\left(\varphi^{m}(x) \varphi^{n}(y)\right)-\rho\left(\varphi^{m}(x)\right) \rho\left(\varphi^{n}(y)\right)
$$


for $m>0$ and $n>0$. The connected form $\rho_{c}$ is an infinitesimal form (that is, $\left.\rho_{c}(1)=0\right)$. For $u$ as above, $\rho_{c}(u)$ is defined as the sum of the connected terms of $\rho(u)$. This terminology is due to the fact that we can define Feynman diagrams to represent $\rho(u)$, and that $\rho_{c}(u)$ is obtained by summing the connected Feynman diagrams present in $\rho(u)$.

Note that the relation $\rho=\mathrm{e}^{\hat{*} \rho_{c}}$ is the analogue of the relation $Z=\mathrm{e}^{W}$ between the partition function and the free energy. As we prove now, the latter is a consequence of the former. The Hamiltonian density of a quantum field theory of local interactions is of the form $H(x)$. Therefore, $\delta H(x)=H(x) \otimes 1+1 \otimes H(x)$ is primitive and, by lemma $5.1, Z=\rho(S)=\mathrm{e}^{W}$, where $W=\rho_{c}(S)$ (see 22 for a detailed proof). This is an extension of the standard relation to the case of a general initial state. As a matter of fact, the coproduct $\delta$ was precisely defined for the Hamiltonian to be a primitive element. This ensures the standard relation between the partition function and the free energy (compare to [5]).

6.2. Example of the $\varphi^{3}$ theory. As we saw in section 2.3 , the two-point Green function for a system described by the interaction Hamiltonian density $u=$ $\varphi^{3}(x)$ is given by the expression:

$$
G(x, y)=\frac{\left\langle 0\left|T\left(\varphi(x) \varphi(y) \mathrm{e}^{-i u}\right)\right| 0\right\rangle}{\left\langle 0\left|T\left(\mathrm{e}^{-i u}\right)\right| 0\right\rangle} .
$$

We recall that the denominator cancels the divergence of the adiabatic switching of the interaction. In graphical terms, the denominator $\left\langle 0\left|T\left(\mathrm{e}^{-i u}\right)\right| 0\right\rangle$ is the sum of all the vacuum Feynman diagrams (i.e. the diagrams that are linked neither to $x$ nor to $y$ ). Another way to obtain a convergent expression is to use the connected Green function $G_{c}(x, y)$ which is the sum of all the connected diagrams in $G(x, y)$.

For a general form, the factorization of the adiabatic divergence is more complex [40, 49, 50] and it holds only for specific initial states [32]. For notational convenience, we do not write the denominator in the definition of the Green functions for a general form and we put

$$
G(x, y)=\rho\left(\varphi(x) \varphi(y) \mathrm{e}^{-i \int_{-\infty}^{\infty} H_{\text {int }}(t)}\right) .
$$

The connected Green function is defined as

$$
G_{c}(x, y)=\rho_{c}\left(\varphi(x) \varphi(y) \mathrm{e}^{-i \int_{-\infty}^{\infty} H_{\text {int }}(t)}\right) .
$$

The term $\int_{-\infty}^{\infty} H_{\text {int }}(t)$ can usually be written $\int \mathrm{d} x P(x)$, where $P(x)$ is a polynomial in $\varphi(x)$. Therefore,

$G(x, y)=\rho(\varphi(x) \varphi(y))+\sum_{n=1}^{\infty} \frac{(-i)^{n}}{n !} \int \mathrm{d} x_{1} \ldots \mathrm{d} x_{n} \rho\left(\varphi(x) \varphi(y) P\left(x_{1}\right) \ldots P\left(x_{n}\right)\right)$.

For example, in the $\varphi^{3}$ theory, we have $P(x)=\varphi^{3}(x) / 3$ ! and the first terms of the total Green function are

$$
\begin{aligned}
G(x, y)= & \rho(\varphi(x) \varphi(y))-\frac{i}{6} \int \mathrm{d} x_{1} \rho\left(\varphi(x) \varphi(y) \varphi^{3}\left(x_{1}\right)\right) \\
& -\frac{1}{72} \int \mathrm{d} x_{1} \mathrm{~d} x_{2} \rho\left(\varphi(x) \varphi(y) \varphi^{3}\left(x_{1}\right) \varphi^{3}\left(x_{2}\right)\right)+\ldots
\end{aligned}
$$

For notational convenience, we assume that $D_{n}\left(x_{1}, \ldots, x_{n}\right)=r\left(\varphi\left(x_{1}\right) \ldots \varphi\left(x_{n}\right)\right)=$ 0 if $n$ is odd. The connected Green function $G_{c}(x, y)$ is obtained by keeping the connected terms of the total Green function. 
In standard quantum field theory, the expansion to the second order gives us (6) $G_{c}(x, y)=D_{2}(x, y)-\frac{1}{2} \int \mathrm{d} z \mathrm{~d} w D_{2}(x, z) D_{2}(y, w) D_{2}(z, w)^{2}+\ldots$

For a general form, the expansion to the second order gives a more complex result:

(7)

$$
\begin{aligned}
G_{c}(x, y)= & D_{2}(x, y)-\int \mathrm{d} z \mathrm{~d} w\left(\frac{1}{72} D_{8}(x, y, z, z, z, w, w, w)\right. \\
& +\frac{1}{12} D_{2}(x, z) D_{6}(y, z, z, w, w, w) \\
+ & \frac{1}{12} D_{6}(x, z, z, w, w, w) D_{2}(y, z)+\frac{1}{12} D_{6}(x, y, z, w, w, w) D_{2}(z, z) \\
+ & \frac{1}{8} D_{6}(x, y, z, z, w, w) D_{2}(z, w)+\frac{1}{12} D_{4}(x, y, z, z) D_{4}(z, w, w, w) \\
+ & \frac{1}{8} D_{4}(x, y, z, w) D_{4}(z, z, w, w)+\frac{1}{4} D_{4}(x, z, z, w) D_{4}(y, z, w, w) \\
+ & \frac{1}{6} D_{2}(x, z) D_{2}(y, z) D_{4}(z, w, w, w)+\frac{1}{4} D_{2}(x, z) D_{2}(y, w) D_{4}(z, z, w, w) \\
+ & \frac{1}{2} D_{2}(x, z) D_{4}(y, z, w, w) D_{2}(z, w)+\frac{1}{4} D_{2}(x, z) D_{4}(y, z, z, w) D_{2}(w, w) \\
+ & \frac{1}{2} D_{4}(x, z, w, w) D_{2}(y, z) D_{2}(z, w)+\frac{1}{4} D_{4}(x, z, z, w) D_{2}(y, z) D_{2}(w, w) \\
+ & \frac{1}{4} D_{4}(x, y, w, w) D_{2}(z, z) D_{2}(z, w)+\frac{1}{8} D_{4}(x, y, z, w) D_{2}(z, z) D_{2}(w, w) \\
+ & \frac{1}{4} D_{4}(x, y, z, w) D_{2}(z, w)^{2}+\frac{1}{2} D_{2}(x, z) D_{2}(y, z) D_{2}(z, w) D_{2}(w, w) \\
+ & \left.\frac{1}{2} D_{2}(x, z) D_{2}(y, w) D_{2}(z, w)^{2}\right)+\ldots
\end{aligned}
$$

These terms can be given the diagrammatic representation of figure 2 .

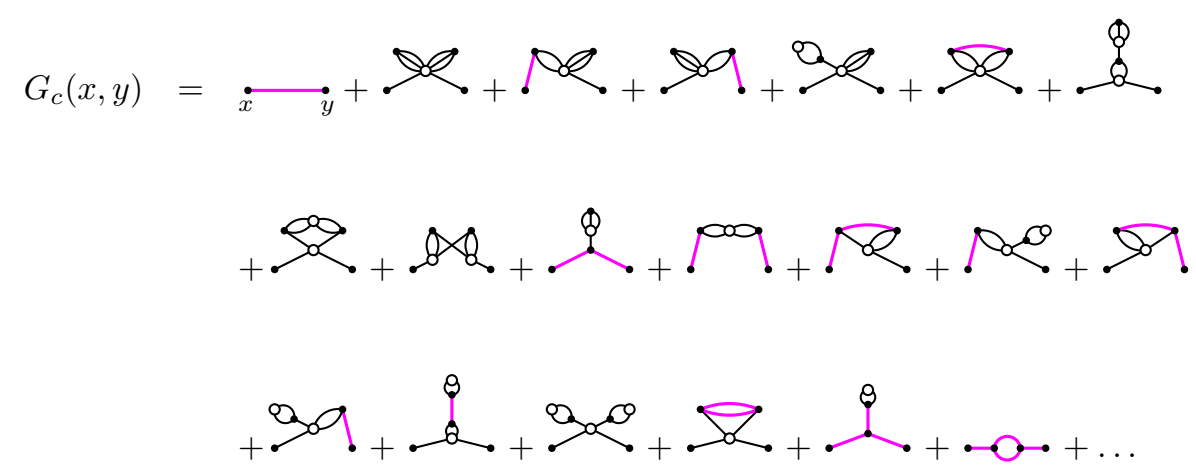

Figure 2. The first few terms of $G_{c}(x, y)$

It is clear from a comparison of equations (6) and (8) that the use of a form $\rho$ which does not come from a quasi-free state increases significantly the combinatorial complexity. As a consequence, little was known about the structure of the connected 
Green functions in the general case, especially as far as one-particle-irreducibility is concerned.

\section{Symmetric functions and derivations}

In [19, 20], Mestre and Oeckl have proposed a powerful Hopf algebraic tool to generate 1PI diagrams. The forthcoming developments of the present article aim at extending their work to correlated systems, and at describing in this general setting the decomposition of connected Green functions into one-particle irreducible Green functions.

In order to do so, the language of functional derivatives proves to be a very convenient framework. This leads naturally to the study of Hopf algebra derivations, which is the main topic of the present section. Namely, we describe some aspects of the algebra of symmetric functions in the Hopf algebraic setting and prove that this algebra has universal properties with respect to derivations acting on commutative Hopf algebras. We will show later that this formalism nicely encodes the properties of the generalized $n$-point propagators associated to arbitrary states, as described above. We do not seek the utmost generality in our constructions, but mention that they can be easily extended to more general (e.g. noncommutative) situations.

7.1. Definition of the derivations $A_{m}$. Let us consider, once again, the Hopf algebra $U=S(V)$. Two products arise therefore in $S(U)=S(S(V))$ : the symmetric product in $S(V)$, denoted by juxtaposition, and the symmetric product in $S(U)$, denoted from now on by $\vee$ to avoid any confusion.

To a unital form $\rho$ on $U=S(V)$, we associate its convolution logarithm $r$ and, for $\varphi\left(x_{1}\right) \ldots \varphi\left(x_{m}\right) \in U$, we recall that $D_{m}\left(x_{1}, \ldots, x_{m}\right)=r\left(\varphi\left(x_{1}\right) \ldots \varphi\left(x_{m}\right)\right)$, so that the generalized propagators $D_{m}$ are symmetric functions of their arguments.

Recall that $V$ is spanned by the symbols $\varphi(x)$, where $x$ runs over points in $\mathbb{R}^{d}$. We choose an arbitrary total order on the points in $\mathbb{R}^{d}$, for example the lexicographical order on the $d$-tuples of coordinates. The operators $A_{m-1}: U \rightarrow$ $S^{m}(U)$ are then defined by

$$
\begin{aligned}
A_{m-1}(u) & =\sum_{x_{1}<\ldots<x_{m}} D_{m}\left(x_{1}, \ldots, x_{m}\right) \frac{\bar{\partial} u_{\{1\}}}{\partial \varphi\left(x_{1}\right)} \vee \cdots \vee \frac{\bar{\partial} u_{\{m\}}}{\partial \varphi\left(x_{m}\right)} \\
& =\frac{1}{m !} \sum_{x_{1}, \ldots, x_{m}} D_{m}\left(x_{1}, \ldots, x_{m}\right) \frac{\bar{\partial} u_{\{1\}}}{\partial \varphi\left(x_{1}\right)} \vee \cdots \vee \frac{\bar{\partial} u_{\{m\}}}{\partial \varphi\left(x_{m}\right)},
\end{aligned}
$$

where $\frac{\bar{\partial} u}{\partial \varphi(x)}$ is defined by the following (topologically motivated) rule:

- $\frac{\bar{\partial} u}{\partial \varphi(x)}:=\frac{\partial u}{\partial \varphi(x)}$ if $u$ is not linear in $\varphi(x)$ or if $\frac{\partial u}{\partial \varphi(x)}$ is a scalar.

- $\frac{\bar{\partial} u}{\partial \varphi(x)}:=0$ otherwise.

These operators on $U$ extend uniquely to derivations on $S(U)$, still written $A_{m-1}$, so that:

$$
A_{m-1}: S^{k}(U) \longrightarrow S^{k+m-1}(U)
$$

(recall, for completeness sake, that a derivation $D$ satisfies the Leibniz rule, $D(x y)=$ $D(x) y+x D(y)$, so that the action of a derivation on $S(U)$ follows by induction on the degrees from the knowledge of its action on $U$ ). Notice also that, by definition of $\delta$, the terms with $x_{i}=x_{j}$ vanish in the sum of eq. (9). 
In practice, forcing the null value in the linear case (when $u$ is not a scalar multiple of $\varphi(x)$ ) will permit to avoid the creation of topologically disconnected graphs. We are particularly grateful to Achim Randelhoff who pointed out to us this property and allowed us to correct an error in a previous version of this article. The meaning of this observation should become clear later on, but the reader may immediately understand its implications by comparing the action of $A_{1}^{2}$ on $\varphi(x) \varphi(y) \varphi(z)^{2}$ with the action of the derivation $\hat{A}_{1}^{2}$, where the action of $\hat{A}_{m-1}$ is defined on $U$ by:

$$
\hat{A}_{m-1}(u)=\sum_{x_{1}<\ldots<x_{m}} D_{m}\left(x_{1}, \ldots, x_{m}\right) \frac{\partial u_{\{1\}}}{\partial \varphi\left(x_{1}\right)} \vee \cdots \vee \frac{\partial u_{\{m\}}}{\partial \varphi\left(x_{m}\right)} .
$$

An important point for our forthcoming developments is that the $A_{i}$ commute. This follows from the Schwarz commutation rules for derivatives and from the definition of the disconnecting coproduct $\delta$. Graphically, these operations will allow us to construct inductively 1 PI graphs (with a given set of vertices and with given multiplicities), and their algebraic properties will allow us to enumerate these graphs. This will be the purpose of the next section, whereas the following subsections introduce the abstract Hopf algebraic framework suited for this enumeration.

7.2. The Hopf algebra of symmetric functions. Let $X=\left\{x_{1} \ldots x_{n} \ldots\right\}$ be a countable alphabet, and $\mathbb{C}[[X]]$ the algebra of formal power series over $X$. The group $\mathcal{S}_{\infty}=\lim \mathcal{S}_{n}$ acts on $\mathbb{C}[[X]]$ by permutation of the letters of $X$; the algebra of symmetric functions $\mathbb{S} y m$ is the subalgebra of $\mathcal{S}_{\infty}$-invariant series in $\mathbb{C}[[X]]$.

The algebra $\mathbb{S} y m$ is (up to completion with respect to the filtration induced by the grading of symmetric polynomials by their degree) a free commutative algebra over various families of generators. For our purposes, the most interesting ones are the families of power sums symmetric functions and complete symmetric functions associated respectively to the series

$$
\text { P. }:=\sum_{k \in \mathbb{N}} P_{k}:=1+\sum_{i \in \mathbb{N}} \frac{x_{i}}{1-x_{i}}
$$

and

$$
\text { C. }:=\sum_{k \in \mathbb{N}} S_{k}:=\prod_{i \in \mathbb{N}} \frac{1}{1-x_{i}} .
$$

In view of our forthcoming computations, it is actually convenient to work with an extension of $\mathbb{S} y m, q \mathbb{S} y m$ : we write $Q_{k}$ for $\frac{P_{k}}{k}, k \geq 1, Q_{0}:=q$ and $\mathbf{Q}_{\bullet}:=\sum_{k \in \mathbb{N}} Q_{k}$, where $q$ stands for an additional free variable. The series are related by the familiar Newton-type identity: $\mathbf{C}_{\bullet}=e^{\mathbf{Q}_{\bullet}-Q_{0}}$. We write $\mathbf{S}_{\bullet}$ for the $q$-series $\mathbf{S}_{\bullet}:=e^{Q_{0}} \mathbf{C}_{\bullet}=$ $e^{\mathbf{Q} \bullet}$

The algebra $q \mathbb{S} y m$ carries a natural notion of grading (by the degrees of symmetric polynomials, with $\operatorname{deg}\left(Q_{n}\right)=n$ ), but it is convenient, for our purposes, and for reasons that will become clear later, to introduce an extra "auxiliary" grading by considering the family of the $Q_{k}$ as a family of generators of $\mathbb{S} y m$ of auxiliary degree 1. This is best explained through an example: $Q_{0}^{2} Q_{3} Q_{5} Q_{9}$ is of degree 17 and of auxiliary degree 5 . The auxiliary degree is indicated with a superscript (whereas the degree is indicated by a subscript), so that, for example, the component of 
degree $n$ and auxiliary degree $k$ in $\mathbf{S}$. is given by:

$$
\mathbf{S}_{n}^{k}=\sum_{\alpha} \frac{Q_{0}^{\alpha_{0}}}{\alpha_{0} !} \frac{Q_{1}^{\alpha_{1}}}{\alpha_{1} !} \ldots \frac{Q_{n}^{\alpha_{n}}}{\alpha_{n} !}
$$

where the sum runs over all $(n+1)$-tuples of integers $\alpha=\left(\alpha_{0}, \ldots, \alpha_{n}\right)$ with $\alpha_{0}+\cdots+$ $\alpha_{n}=k$ and $\alpha_{1}+2 \alpha_{2}+\cdots+n \alpha_{n}=n$. Notice that we distinguish carefully between $\mathbf{S}_{n}^{k}$ and $S_{n}^{k}$, the latter standing for the $k$-th power of $S_{n}$. The following examples will be useful in the sequel: $\mathbf{S}_{n}^{0}=\delta_{n, 0} 1, \mathbf{S}_{0}^{k}=Q_{0}^{k} / k !, \mathbf{S}_{n}^{1}=Q_{n}, \mathbf{S}_{1}^{k}=Q_{0}^{k-1} Q_{1} /(k-1)$ !, $\mathbf{S}_{2}^{2}=Q_{0} Q_{2}+Q_{1}^{2} / 2$.

Generating series are a useful tool to handle computations with the $\mathbf{Q}_{n}^{k}$ and the $\mathbf{S}_{n}^{k}$. Consider for example the series $\mathbf{S}_{\bullet}(a+b):=e^{(a+b) \mathbf{Q}_{\bullet}}$ : its expansion as a series in the variables $a$ and $b$ yields:

Proposition 7.1. We have, for all $k, l \geq 0$ :

$$
\left(\begin{array}{c}
k+l \\
k
\end{array}\right) \mathbf{S}_{n}^{k+l}=\sum_{m=0}^{n} \mathbf{S}_{m}^{k} \mathbf{S}_{n-m}^{l}
$$

In particular

$$
\mathbf{S}_{n}^{k}=\frac{1}{k} \sum_{m=0}^{n} Q_{m} \mathbf{S}_{n-m}^{k-1} .
$$

The Hopf algebraic properties of symmetric functions were recently exploited with great profit by Fauser and coll. [51, 52. Similarly, we put a simple Hopf algebraic structure on $q \mathbb{S} y m$ uniquely defined by requiring the power sums symmetric functions (i.e. $Q_{n}$ for $n>0$ ) to form, together with $Q_{0}$, a series of primitive elements or, equivalently, by requiring the series $\mathbf{S}$ • to be a group-like element. In other terms, the coproduct $\Delta$ on $q \mathbb{S} y m$ is fully specified by requiring that $\Delta\left(Q_{n}\right):=Q_{n} \otimes 1+1 \otimes Q_{n}, n \geq 0$. In particular, the coproduct is compatible with the two graduations. When expliciting this property in $\mathbf{S}_{n}^{k}$, we get:

Proposition 7.2. The coproduct of $\mathbf{S}_{n}^{k}$ is

$$
\Delta \mathbf{S}_{n}^{k}=\sum_{m=0}^{n} \sum_{i=0}^{k} \mathbf{S}_{m}^{i} \otimes \mathbf{S}_{n-m}^{k-i}
$$

and its iterated coproduct is

$$
\Delta^{p-1} \mathbf{S}_{n}^{k}=\sum_{\substack{n_{1}+\cdots+n_{p}=n \\ k_{1}+\cdots+k_{p}=k}} \mathbf{S}_{n_{1}}^{k_{1}} \otimes \cdots \otimes \mathbf{S}_{n_{p}}^{k_{p}},
$$

Note that propositions 7.1 and 7.2 still hold if the variables $Q_{n}$ do not commute.

7.3. On Hopf algebra derivations. Let $H=\bigoplus_{n \in \mathbb{N}} H_{n}$ be an arbitrary connected graded commutative Hopf algebra and $A_{0}, A_{1}, \ldots, A_{n}, \ldots$ an arbitrary sequence of derivations on $H$ with degrees $0,1, \ldots, n, \ldots$ That is, for any $p$, the restriction of $A_{n}$ to $H_{p}$ is a linear map from $H_{p}$ to $H_{p+n}$, and $A_{n}$ satisfies the Leibniz rule: for any $h, l$ in $H, A_{n}(h \cdot l)=A_{n}(h) \cdot l+h \cdot A_{n}(l)$. We also assume that the $A_{n}$ commute, so that the $A_{n}$ generate a commutative subalgebra $\mathcal{D}$ of $\operatorname{End}(H)$ (for the composition of maps). Of course, we have in mind the particular derivations $A_{m}$ acting on $S(U)$, but the following results hold in full generality. 
There is therefore, since $q \mathbb{S} y m$ is free over the $Q_{n}$, a universal algebra map $\beta$ from $q \mathbb{S} y m$ to $\operatorname{End}(H)$ obtained by mapping $Q_{n}$ to $A_{n}$. We write $\mathbf{L}_{\bullet}$ for the image of $\mathbf{S}$. under this map, and $L_{n}^{k}$ for the image of $\mathbf{S}_{n}^{k}$. Note that, for any $p, L_{n}^{k}$ maps $H_{p}$ to $H_{p+n}$. Of course, the identities that hold in $q \mathbb{S} y m$ for the variables $Q_{n}$ and $\mathbf{S}_{n}^{k}$ also hold in $\operatorname{End}(H)$ for the variables $A_{n}$ and $L_{n}^{k}$. More surprisingly however, the coalgebra structure of $q \mathbb{S} y m$ reflects the action of $\mathcal{D}$ on $H$. We refer to [53, 16] for similar phenomena occurring in the study of Lie idempotents and renormalization in perturbative QFT.

Proposition 7.3. We have, for any $X \in q \mathbb{S} y m$ and any $h, h^{\prime} \in H$ :

$$
\beta(X)\left(h h^{\prime}\right)=\beta\left(X_{(1)}\right)(h) \beta\left(X_{(2)}\right)\left(h^{\prime}\right)
$$

where $X_{(1)} \otimes X_{(2)}$ stands, as usual, for the coproduct of $X$ in $q \mathbb{S} y m$.

The identity can be generalized by a straightforward recursion to compute $\beta(X)\left(h_{1} \ldots h_{n}\right)$. Notice first that the identity in the Proposition is obvious when $X$ is a $Q_{n}$, since $\beta\left(Q_{n}\right)=A_{n}$ is, by hypothesis, a derivation. Now, assume that for $X$ and $Y$ in $q \mathbb{S} y m$ and arbitrary $h, h^{\prime}, l, l^{\prime} \in H$ the above formula holds, that is:

$$
\beta(X)\left(h h^{\prime}\right)=\beta\left(X_{(1)}\right)(h) \beta\left(X_{(2)}\right)\left(h^{\prime}\right), \beta(Y)\left(l l^{\prime}\right)=\beta\left(Y_{(1)}\right)(l) \beta\left(Y_{(2)}\right)\left(l^{\prime}\right) .
$$

It follows that:

$$
\begin{aligned}
\beta(Y) \circ \beta(X)\left(h h^{\prime}\right) & =\beta(Y)\left(\beta\left(X_{(1)}\right)(h) \beta\left(X_{(2)}\right)\left(h^{\prime}\right)\right) \\
& =\beta\left(Y_{(1)}\right) \circ \beta\left(X_{(1)}\right)(h) \beta\left(Y_{(2)}\right) \circ \beta\left(X_{(2)}\right)\left(h^{\prime}\right) \\
& =\beta\left((Y X)_{(1)}\right)(h) \beta\left((Y X)_{(2)}\right)\left(h^{\prime}\right) .
\end{aligned}
$$

In other terms, if two elements in q $\mathbb{S} y m$ satisfy the identity in the Proposition, their product also satisfies the identity. Since the $Q_{n}$ satisfy the identity, and since their products span $q \mathbb{S} y m$, the Proposition follows.

Let us consider the particular example of $S(U)$ and of the derivations $A_{m-1}$. Let us write $A_{m-1}(u)=u_{m-1,1} \vee \cdots \vee u_{m-1, m}$, with an enhanced Sweedler-type notation for the action of $A_{m-1}$ from $U$ to $S^{m}(U)$. We get:

$$
\begin{aligned}
L_{n}^{k}(u) & =\frac{1}{k} \sum_{m=1}^{n+1} L_{n-m+1}^{k-1} A_{m-1}(u) \\
& =\frac{1}{k} \sum_{m=1}^{n+1} \sum_{\substack{k_{1}+\cdots+k_{m}=k-1 \\
n_{1}+\cdots+n_{m}=n-m+1}} L_{n_{1}}^{k_{1}}\left(u_{m-1,1}\right) \vee \cdots \vee L_{n_{m}}^{k_{m}}\left(u_{m-1, m}\right),
\end{aligned}
$$

where $\vee$ is the symmetric product in $S(U)$. This is a generalization of lemma 13 in [19] and of proposition 15 in [20] where Mestre and Oeckl studied the case where $A_{n}=0$ for $n \neq 0,1$. Note that our notation is different from theirs.

\section{One-particle irreducible decompositions}

We consider now the derivation of an explicit decomposition of a connected Green function into 1PI Green functions for a general state. 
As we have just noticed, the map $Q_{m} \mapsto A_{m}$ enables us to define $L_{n}^{k}$ acting on $S(U)=S(S(V))$. For example $L_{0}^{0}(u)=u, L_{m}^{1}(u)=A_{m}(u)$ and

$$
\begin{aligned}
L_{0}^{2}(u)= & \frac{1}{2} \sum_{x_{1}, x_{2}} D_{1}\left(x_{1}\right) D_{1}\left(x_{2}\right) \frac{\bar{\partial}^{2} u}{\partial \varphi\left(x_{1}\right) \partial \varphi\left(x_{2}\right)}, \\
L_{1}^{2}(u)= & \sum_{x_{1}, x_{2}, x_{3}} D_{2}\left(x_{1}, x_{2}\right) D_{1}\left(x_{3}\right) \frac{\bar{\partial} u_{\{1\}}}{\partial \varphi\left(x_{1}\right)} \vee \frac{\bar{\partial}^{2} u_{\{2\}}}{\partial \varphi\left(x_{2}\right) \partial \varphi\left(x_{3}\right)}, \\
L_{2}^{2}(u)= & \frac{1}{2} \sum_{x_{1}, x_{2}, x_{3}, x_{4}} D_{2}\left(x_{1}, x_{2}\right) D_{2}\left(x_{3}, x_{4}\right) \frac{\bar{\partial} u_{\{1\}}}{\partial \varphi\left(x_{1}\right)} \vee \frac{\bar{\partial}^{2} u_{\{2\}}}{\partial \varphi\left(x_{2}\right) \partial \varphi\left(x_{3}\right)} \vee \frac{\bar{\partial} u_{\{3\}}}{\partial \varphi\left(x_{4}\right)} \\
& +\frac{1}{2} \sum_{x_{1}, x_{2}, x_{3}, x_{4}} D_{3}\left(x_{1}, x_{2}, x_{3}\right) D_{1}\left(x_{4}\right) \frac{\bar{\partial} u_{\{1\}}}{\partial \varphi\left(x_{1}\right)} \vee \frac{\bar{\partial} u_{\{2\}}}{\partial \varphi\left(x_{2}\right)} \vee \frac{\bar{\partial}^{2} u_{\{3\}}}{\partial \varphi\left(x_{3}\right) \partial \varphi\left(x_{4}\right)}, \\
L_{1}^{3}(u)= & \frac{1}{2} \sum_{x_{1}, x_{2}, x_{3}, x_{4}} D_{1}\left(x_{1}\right) D_{1}\left(x_{2}\right) D_{2}\left(x_{3}, x_{4}\right) \frac{\bar{\partial}^{2} u_{\{1\}}}{\partial \varphi\left(x_{1}\right) \partial \varphi\left(x_{3}\right)} \vee \frac{\bar{\partial}^{2} u_{\{2\}}}{\partial \varphi\left(x_{2}\right) \partial \varphi\left(x_{4}\right)} \\
& +\frac{1}{2} \sum_{x_{1}, x_{2}, x_{3}, x_{4}} D_{1}\left(x_{1}\right) D_{1}\left(x_{2}\right) D_{2}\left(x_{3}, x_{4}\right) \frac{\bar{\partial}^{3} u_{\{1\}}}{\partial \varphi\left(x_{1}\right) \partial \varphi\left(x_{2}\right) \partial \varphi\left(x_{3}\right)} \vee \frac{\bar{\partial} u_{\{2\}}}{\partial \varphi\left(x_{4}\right)} .
\end{aligned}
$$

8.1. A tree interpretation. The operator $L_{n}^{k}$ can be written as a sum over all the bipartite trees with $k$ white vertices and $n+1$ black vertices. This description in terms of trees is important because, in standard QFT, a connected Green function can also be described as a tree of 1PI Green functions - a description we want to extend to the case of initial correlations. To give a more precise relation between $L_{n}^{k}$ and bipartite trees, we consider the expression for $L_{n}^{k}$ in terms of partitions $\alpha$ :

$$
L_{n}^{k}=\sum_{\alpha} \frac{A_{0}^{\alpha_{0}}}{\alpha_{0} !} \ldots \frac{A_{n}^{\alpha_{n}}}{\alpha_{n} !}
$$

where the sum runs over the sequences $\alpha$ of nonnegative integers with $\alpha_{0}+\cdots+\alpha_{n}=$ $k$ and $\alpha_{1}+\cdots+n \alpha_{n}=n$. The monomial corresponding to a given $\alpha$ is represented by the sum of all bipartite trees with $k$ white vertices and $n+1$ black vertices, such that $\alpha_{i}$ white vertices have valency $i+1$, for $i=1, \ldots, n$. 
The terms of lowest degrees are

$$
\begin{aligned}
& L_{0}^{0}=\mathrm{id}=\bullet, \\
& L_{0}^{1}=A_{0}=\bullet \bullet \text {, } \\
& L_{0}^{2}=\frac{1}{2 !} A_{0}^{2}=\bullet \bullet-\circ, \\
& L_{1}^{1}=A_{1}=\bullet \bullet, \\
& L_{0}^{3}=\frac{1}{3 !} A_{0}^{3}=\text {. } \\
& L_{1}^{2}=A_{0} A_{1}=\bullet \bullet \bullet-\circ, \\
& L_{2}^{1}=A_{2}=\stackrel{i}{\circ} \text {, } \\
& L_{0}^{4}=\frac{1}{4 !} A_{0}^{4}=\boldsymbol{X}_{0}^{\circ} \text {, } \\
& L_{2}^{2}=A_{0} A_{2}+\frac{1}{2} A_{1}^{2}=: \bullet \bullet \bullet+\cdots \cdots \cdots \\
& L_{1}^{3}=\frac{1}{2} A_{0}^{2} A_{1}=\cdots \bullet-\bullet+>\bullet \bullet \bullet, \\
& L_{3}^{1}=A_{3}= \\
& L_{0}^{5}=\frac{1}{5 !} A_{0}^{5}=8 \text {, } \\
& L_{3}^{2}=A_{0} A_{3}+A_{1} A_{2}=: \bullet \bullet-\bullet+\bullet \bullet \bullet,
\end{aligned}
$$

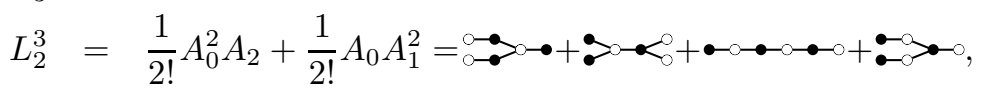

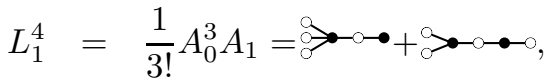

$$
\begin{aligned}
& L_{4}^{1}=A_{4}=\stackrel{i}{\circ} \text {. }
\end{aligned}
$$

To calculate the value of a tree of $L_{n}^{k}$ : (i) Associate to each of the $k+n$ edges a variable $x_{i}$, with $i=1, \ldots, k+n$. (ii) To each white vertex $v$, associate the factor $D_{m}\left(x_{i_{1}}, \ldots, x_{i_{m}}\right)$, where $m$ is the valency of $v$ and $x_{i_{1}}, \ldots, x_{i_{m}}$ are the variables associated to the edges incident to $v$. (iii) There are $n+1$ black vertices. Split $u$ into $n+1$ parts by $\delta^{n} u=u_{\{1\}} \otimes \cdots \otimes u_{\{n+1\}}$. Number the black vertices from 1 to $n+1$ and to vertex $\ell$ associate the factor

$$
\frac{\bar{\partial}^{m} u_{\{\ell\}}}{\partial \varphi\left(x_{i_{1}}\right) \ldots \partial \varphi\left(x_{i_{\ell}}\right)},
$$

where $x_{i_{1}}, \ldots, x_{i_{\ell}}$ are the variables associated to the edges incident to the black vertex number $\ell$. (iv) Multiply the factors corresponding to the black vertices with the product $\vee$ in $S(U)$. (v) Divide the resulting value by the order of the symmetry group of the tree.

8.2. The 1PI components of forms. The last step before we can write a connected form in terms of $1 \mathrm{PI}$ forms is to give a reasonable definition of what is the 1PI component of a form, similarly to the definition of the connected components of forms. Several definitions are possible. The simplest one was proposed by Hall [4] and has recently provided detailed structural results [54]. Here we consider a definition which is strictly more general than Hall's and that leads to an interesting structure. In a graph, it is easy to describe what we mean by cutting a line or a set 
of lines; this approach leads, in classical QFT (with 2-point Feynman propagators) to the definition of 1PI Feynman diagrams as connected diagrams that are still connected when an arbitrary propagator line is cut. We propose to generalize the notion by replacing the Feynman form (that is, the classical case where only 2-point Feynman propagators are considered) by an arbitrary unital form.

Our approach is rooted in the Hopf algebraic picture of QFT. Notice however that our constructions could be translated mutatis mutandis in the language of functional derivatives. For example, the derivatives $\frac{\partial}{\partial \varphi(x)}$ that we have used in the definition of the operators $A_{i}$ were defined as usual derivatives (in the polynomial algebra over the symbols $\varphi(x)$ ) but could be understood alternatively as functional derivatives. The same observation holds for our forthcoming constructions.

In proposition 6.1, for any $u=\varphi^{n_{1}}\left(x_{1}\right) \ldots \varphi^{n_{k}}\left(x_{k}\right)$, we have expanded $\rho(u)$ as a linear combination of terms such as $r\left(\varphi^{n_{1}^{1}}\left(x_{1}\right) \ldots \varphi^{n_{k}^{1}}\left(x_{k}\right)\right) \ldots r\left(\varphi^{n_{1}^{l}}\left(x_{1}\right) \ldots \varphi^{n_{k}^{l}}\left(x_{k}\right)\right)$. Let us consider a connected term

$$
t:=r\left(\varphi^{n_{1}^{1}}\left(x_{1}\right) \ldots \varphi^{n_{k}^{1}}\left(x_{k}\right)\right) \ldots r\left(\varphi^{n_{1}^{l}}\left(x_{1}\right) \ldots \varphi^{n_{k}^{l}}\left(x_{k}\right)\right)
$$

in $\mathrm{e}^{* r}(u)$.

Definition 8.1. The connected term $t$ is said to be one-particle reducible if and only if, there exists $i \in\{1, \ldots, l\}$ and $\left\{i_{1}, \ldots, i_{p}\right\} \subset\{1, \ldots, k\}$ such that

(1) $\varphi^{n_{1}^{i}}\left(x_{1}\right) \ldots \varphi^{n_{k}^{i}}\left(x_{k}\right)=\varphi\left(x_{i_{1}}\right) \ldots \varphi\left(x_{i_{p}}\right)$

(2) furthermore, in the remaining part of $t$,

$$
\begin{aligned}
& r\left(\varphi^{n_{1}^{1}}\left(x_{1}\right) \ldots \varphi^{n_{k}^{1}}\left(x_{k}\right)\right) \ldots r\left(\varphi^{n_{1}^{i-1}}\left(x_{1}\right) \ldots \varphi^{n_{k}^{i-1}}\left(x_{k}\right)\right) \\
& \quad r\left(\varphi^{n_{1}^{i+1}}\left(x_{1}\right) \ldots \varphi^{n_{k}^{i+1}}\left(x_{k}\right)\right) \ldots r\left(\varphi^{n_{1}^{l}}\left(x_{1}\right) \ldots \varphi^{n_{k}^{l}}\left(x_{k}\right)\right)
\end{aligned}
$$

the connected components of $x_{i_{1}}, \ldots, x_{i_{p}}$ are either empty or pairwise disjoint.

A connected term that is not one-particle reducible is said to be one-particle irreducible (1PI).

For example, $r\left(\varphi\left(x_{1}\right) \varphi\left(x_{2}\right)\right)^{2}$ (a loop constructed out of two two-point propagators) is 1PI (in our situation, and also in the usual picture), and so is

$$
r\left(\varphi\left(x_{1}\right) \varphi\left(x_{2}\right)\right) r\left(\varphi\left(x_{1}\right) \varphi\left(x_{3}\right)\right) r\left(\varphi\left(x_{1}\right) \varphi\left(x_{2}\right) \varphi\left(x_{3}\right)\right)
$$

whereas

$$
r\left(\varphi\left(x_{1}\right) \varphi\left(x_{2}\right)\right) r\left(\varphi\left(x_{1}\right) \varphi\left(x_{3}\right)\right) r\left(\varphi\left(x_{1}\right) \varphi\left(x_{2}\right) \varphi\left(x_{4}\right)\right)
$$

or

are not.

$$
r\left(\varphi\left(x_{1}\right) \varphi\left(x_{2}\right)\right) r\left(\varphi\left(x_{1}\right) \varphi^{2}\left(x_{3}\right)\right) r\left(\varphi\left(x_{2}\right) \varphi^{2}\left(x_{4}\right)\right)
$$

Definition 8.2. The $1 P I$ component $\rho_{I}$ of a form is the sum of all the 1 PI terms in the connected component of $\rho$.

Recall that forms, their connected components, and also their 1PI components are linear maps from $U=S(V)$ to $\mathbb{C}$. However, any such linear map $l$ can be uniquely extended to a multiplicative map, still written $l$ from $S(U)$ to $\mathbb{C}$ (that is, to a character of the algebra $S(U)$, in the algebraic terminology). Concretely, for $u_{1}, \ldots, u_{n} \in U, l\left(u_{1} \vee \cdots \vee u_{n}\right):=l\left(u_{1}\right) \ldots l\left(u_{n}\right)$. In particular, the connected and 1 PI components of forms can be viewed as characters of the algebra $S(U)$, so that, 
for example, an expression such as $\rho_{c} \circ A_{m}$ makes sense as the composition of a derivation of $S(U)$ and a map from $S(U)$ to $\mathbb{C}$.

Let us consider a simple example to illustrate these ideas, namely the structure equation linking connected and 1PI components of forms in the most common picture of pQFT: an interacting theory -say $\varphi^{3}$ - with Feynman diagrams built of 3 -valent interaction vertices and 2-point propagators. A general Feynman diagram can be described as 1PI diagrams connected by $n \in \mathbb{N}$ Feynman propagators satisfying the property that cutting any of these propagators makes the original diagram disconnected. Taking into account the symmetry factor $n$ ! arising from the fact that these Feynman propagators can be cut successively in an arbitrary order results into a structure equation relating the connected and 1PI Green functions. In Hopf algebraic terms:

$$
\rho_{c}=\rho_{I} \circ e^{F}
$$

where $F=A_{1}$ is the derivation of $S(U)$ associated to the Feynman propagator: for all $u_{1}, \ldots, u_{n} \in U=S(V)$,

$$
\begin{aligned}
F\left(u_{1} \vee \cdots \vee u_{n}\right)= & \frac{1}{2} \sum_{x \neq y} \sum_{i \leq n} D_{2}(x, y) u_{1} \vee \cdots \vee u_{i-1} \vee \\
& \left(\frac{\bar{\partial} u_{i,\{1\}}}{\partial \varphi(x)} \vee \frac{\bar{\partial} u_{i,\{2\}}}{\partial \varphi(y)}\right) \vee u_{i+1} \vee \cdots \vee u_{n},
\end{aligned}
$$

with $D_{2}$ the (quasi-)free 2-point Green function.

In the general case, replacing Feynman propagators $D_{2}$ by arbitrary propagators $D_{n}$, doesn't change the general principles of the proof. An arbitrary Feynman diagram for an interacting theory as the ones considered previously in the present section can still be cut into 1PI pieces connected by a family of $n$-point propagators, $n \in N$, in such a way that removing any of these $n$-point propagators splits the original diagram into $n$ connected pieces. For a given $n$, the associated symmetry factor is $p_{n}$ !, where $p_{n}$ is the number of $n$-point propagators in the family. These observations result in a family of structure identities for 1PI diagrams at all orders, and an identity that should be understood as a structure theorem for the perturbative approach to interacting theories.

Theorem 8.1. (Structure of connected forms) For an arbitrary connected form $\rho_{c}$, we have

$$
\rho_{c}=\rho_{I} \circ \mathbf{L}_{\bullet}=\rho_{I} \circ e^{\left(\sum_{n \in \mathbb{N}} A_{n}\right)},
$$

and

$$
\rho_{I}=\rho_{c} \circ e^{\left(-\sum_{n \in \mathbb{N}} A_{n}\right)} .
$$

We remark that the effect of $A_{0}$ is just a shift of the fields: for instance $\mathrm{e}^{A_{0}}\left(\varphi^{n}(x)\right)=\left(\varphi(x)+D_{1}(x)\right)^{n}$. For $u=\varphi^{n_{1}}\left(x_{1}\right) \ldots \varphi^{n_{p}}\left(x_{p}\right)$, we have $A_{m}(u)=0$ if $m \geq p$ because $A_{m}$ splits $u$ into $m+1$ pieces and the coordinates $x_{i}$ of these pieces must all be different. More generally, $L_{n}^{k}(u)$ vanishes if $n \geq p$. Because of the trivial effect of $A_{0}$ we put $A_{0}=0$ and we get

$$
\rho_{c}(u)=\rho_{I}(u)+\sum_{n=1}^{p-1} \sum_{k=1}^{n_{1}+\cdots+n_{p}} \rho_{I}\left(L_{n}^{k}(u)\right) .
$$


In particular, $\rho_{c}\left(\varphi^{n}(x)\right)=\rho_{I}\left(\varphi^{n}(x)\right)$ and, for $x \neq y$,

$$
\rho_{c}\left(\varphi^{n}(x) \varphi^{m}(y)\right)=\rho_{I}\left(\varphi^{n}(x) \varphi^{m}(y)\right)+m n D_{2}(x, y) \rho_{I}\left(\varphi^{n-1}(x)\right) \rho_{I}\left(\varphi^{m-1}(y)\right) .
$$

The first equation of theorem 8.1 describes the connected Green functions in terms of 1PI Green functions. It is an extension to general states of the standard QFT result and of a theorem by Mestre and Oeckl [20]. The second equation is new even in the QFT context: it describes the 1PI Green functions as a linear combination of products of connected Green functions. In QFT, 1PI Green functions are expressed in terms of amputated connected Green functions. Here, we do not amputate the Green functions (this is not allowed for a general state because parts of the Green functions belong to the kernel of the differential operator used in the equation of motion of the free field).

The consequences of these identities for the QFT of interacting systems, and the fine study of connected and 1PI amplitudes are postponed to further work.

\section{Conclusion}

In this paper, we developed mathematical tools to extend the relation between connected Green functions and 1PI Green functions from the case of a quasi-free ground state to the case of a general state. Our main result is the structure of connected forms described by theorem 8.1. This work can be extended in two directions. On the physical side, the main structure identity can be used to derive resummation theorems that generalize Friedberg's formulas [55]. On the mathematical side, many of our results can be extended to the case of non commuting variables.

\section{Acknowledgements}

This work originated as a part of a long term joint project on the combinatorics of QFT with Alessandra Frabetti. We thank her very warmly: without the many intense and inspiring discussions that we had with her, this paper would not exist.

We thank Raymond Stora for numerous fruitful comments on a previous version of the manuscript. We also thank warmly Achim Randelhoff who pointed out to us that the definition of the derivations $A_{m-1}$ in a previous version of this article was not suited to their later application to 1-particle irreducibility.

We are grateful to Muriel Livernet for an inspiring remark about the relation between Ref. [19] and one of her works [56] and a proof that some results of Ref. 19] are valid in a general algebraic setting. The help of Jean-Louis Loday is gratefully acknowledged by Ch. B., who is also grateful to John Challifour for sending a copy of his unpublished paper with Wightman.

\section{References}

[1] D. W. Robinson. The ground state of the Bose gas. Commun. Math. Phys., 1:159-74, 1965.

[2] B. S. Kay and R. M. Wald. Theorems on the uniqueness and thermal properties of stationary, nonsingular, quasifree states on spacetimes with a bifurcate Killing horizon. Phys. Repts., 207:49-136, 1991.

[3] B. S. Kay. Sufficient conditions for quasifree states and an improved uniqueness theorem for quantum fields on space-times with horizons. J. Math. Phys., 34:4519-39, 1993.

[4] A. G. Hall. Non-equilibrium Green functions: Generalized Wick's theorem and diagrammatic perturbation theory with initial correlations. J. Phys. A: Math. Gen., 8:214-25, 1975.

[5] S.H. Djah, H. Gottschalk, and H. Ouerdiane. Feynman graph representation of the perturbation series for general functional measures. J. Funct. Anal., 227:153-187, 2005. 
[6] D. Ruelle. Statistical Mechanics, Rigorous Results. W.A. Benjamin Inc., New York, 1969.

[7] H. J. Borchers. Algebraic aspects of Wightman quantum field theory. In H. Araki, editor, International Symposium on Mathematical Problems in Theoretical Physics, volume 39 of Lecture Notes in Physics, pages 283-92, Berlin, 1975. Springer.

[8] R. Stora. Lagrangian field theory. In C. DeWitt and C. Itzykson, editors, Particle Physics Les Houches 1971, pages 1-79, New York, 1973. Gordon and Breach.

[9] A. S. Wightman and J. L. Challifour. On the existence and properties of a two-dimensional quantum field. Unpublished, 69 pages, 1970.

[10] W. Kutzelnigg and D. Mukherjee. Normal order and extended Wick theorem for a multiconfiguration reference wave function. J. Chem. Phys., 107:432-49, 1997.

[11] Ch. Brouder, B. Fauser, A. Frabetti, and R. Oeckl. Quantum field theory and Hopf algebra cohomology. J. Phys. A: Math. Gen., 37:5895-927, 2004.

[12] D. Kreimer. On the Hopf algebra structure of perturbative quantum field theory. Adv. Th. Math. Phys., 2:303-34, 1998.

[13] A. Connes and D. Kreimer. Hopf algebras, renormalization and noncommutative geometry. Commun. Math. Phys., 199:203-42, 1998.

[14] A. Connes and D. Kreimer. Renormalization in quantum field theory and the RiemannHilbert problem I: the Hopf algebra structure of graphs and the main theorem. Commun. Math. Phys., 210:249-73, 2000.

[15] A. Connes and D. Kreimer. Renormalization in quantum field theory and the Riemann-Hilbert problem II: the $\beta$ function, diffeomorphisms and the renormalization group. Commun. Math. Phys., 216:215-41, 2001.

[16] K. Ebrahimi-Fard, J. M. Gracia-Bondia, and F. Patras. A Lie theoretical approach to renormalization. Commun. Math. Phys., 276:519-49, 2007.

[17] B. Fauser. On the Hopf algebraic origin of Wick normal-ordering. J. Phys. A: Math. Gen., 34:105-116, 2001.

[18] A.C. Hirshfeld and P. Henselder. Star products and quantum groups in quantum mechanics and field theory. Ann. Phys., 308:311-28, 2003.

[19] A. Mestre and R. Oeckl. Combinatorics of $n$-point functions via Hopf algebra in quantum field theory. J. Math. Phys., 47:052301, 2006.

[20] A. Mestre and R. Oeckl. Generating loop graphs via Hopf algebra in quantum field theory. J. Math. Phys., 47:122302, 2006.

[21] W. D. van Suijlekom. Renormalization of gauge fields: A Hopf algebra approach. Commun. Math. Phys., 276:773-98, 2007.

[22] Ch. Brouder. Quantum field theory meets Hopf algebra. Math. Nachr., 282:1664-90, 2009.

[23] S. A. Joni and G.-C. Rota. Coalgebras and bialgebras in combinatorics. Stud. Appl. Math., 61:93-139, 1979.

[24] Ch. Brouder. Green function hierarchy for open shells. Euro. Phys. Lett., 71:556-62, 2005.

[25] M. Reed and B. Simon. Methods of Modern Mathematical Physics. II Fourier Analysis, Self-adjointness. Academic Press, New York, 1975.

[26] M. Reed and B. Simon. Methods of Modern Mathematical Physics. I Functional Analysis. Academic Press, New York, second edition, 1980.

[27] A. L. Fetter and J. D. Walecka. Quantum Theory of Many-Particle Systems. McGraw-Hill, Boston, 1971.

[28] M. Gell-Mann and F. Low. Bound states in quantum field theory. Phys. Rev., 84:350-4, 1951.

[29] G. Nenciu and G. Rasche. Adiabatic theorem and Gell-Mann-Low formula. Helv. Phys. Acta, 62:372-88, 1989.

[30] Ch. Brouder, G. Stoltz, and G. Panati. Adiabatic approximation, Gell-Mann and Low theorem and degeneracies: A pedagogical example. Phys. Rev. A, 78:042102, 2008.

[31] Ch. Brouder, G. Panati, and G. Stoltz. Gell-Mann and Low formula for degenerate unperturbed states. Ann. Henri Poincaré, 10:1285-309, 2010.

[32] Ch. Brouder, G. Panati, and G. Stoltz. The many-body Green function of degenerate systems. Phys. Rev. Lett., 103:230401, 2009.

[33] Ch. Brouder and F. Patras. Hyperoctahedral Chen calculus for effective Hamiltonians. J. Algebra 322:4105-4120, 2009.

[34] Ch. Brouder, Â. Mestre and F. Patras Tree expansion in time-dependent perturbation theory J. Math. Phys. 51, 072104, 2010. 
[35] G. Onida, L. Reining, and A. Rubio. Electronic excitations: density-functional versus manybody Green's-function approaches. Rev. Mod. Phys., 74:601-59, 2002.

[36] E. K. U. Gross, E. Runge, and O. Heinonen. Many-Particle Theory. Adam Hilger, Bristol, 1991.

[37] K.-C. Chou, Z.-B. Su, B.-L. Hao, and L. Yu. Equilibrium and nonequilibrium formalisms made unified. Phys. Repts., 118:1-131, 1985.

[38] R. Yaris and H. S. Taylor. Many-body Green's function method for electron scattering from open-shell systems. Phys. Rev. A, 12:1751-9, 1975.

[39] C. Bloch and J. Horowitz. Sur le détermination des premiers états d'un système de fermions dans le cas dégénéré. Nucl. Phys., 8:91-105, 1958.

[40] T. Morita. Perturbation theory for degenerate problems of many-fermion systems. Prog. Theor. Phys., 29:351-69, 1963.

[41] S. Fujita. Introduction to Non Equilibrium Quantum Statistical Mechanics. Saunders, Philadelphia, 1966.

[42] S. Majid. Foundations of Quantum Group Theory. Cambridge University Press, Cambridge, 1995.

[43] M. E. Sweedler. Hopf Algebras. W. A. Benjamin, New York, 1969.

[44] J.C. Collins. Renormalization. Cambridge University Press, Cambridge, 1984.

[45] H. Scutaru. Transition probabilities between quasifree states. J. Math. Phys., 39:6403-15, 1998.

[46] V. A. Moskalenko, P. Entel, D. F. Digor, L. A. Dohotaru, and R. Citro. A diagram approach to the strong coupling in the single-impurity Anderson model. Theor. Math. Phys., 155:914-35, 2008.

[47] S. Hollands and R. M. Wald. Local Wick polynomials and time ordered products of quantum fields in curved spacetime. Commun. Math. Phys., 223:289-326, 2001.

[48] S. G. Thikhodeev. Relations between many-body Green's functions and correlation functions. Sov. Phys. Doklady, 27:492-3, 1982.

[49] T. T. S. Kuo, S. Y. Lee, and K. F. Ratcliff. A folded-diagram expansion of the model-space effective Hamiltonian. Nucl. Phys. A, 176:65-88, 1971.

[50] A. L. Kitanin. Nonstationary perturbation theory for a degenerate discrete level. Theor. Math. Phys., 25:1224-7, 1975.

[51] B. Fauser and P. D. Jarvis. A Hopf laboratory for symmetric functions. J. Phys. A: Math. Gen., 37:1633-63, 2004.

[52] B. Fauser, P. D. Jarvis, R. C. King, and B. G. Wybourne. New branching rules induced by plethysm. J. Phys. A: Math. Gen., 39:2611-55, 2006.

[53] F. Patras and C. Reutenauer. On Dynkin and Klyachko idempotents in graded bialgebras. Adv. Appl. Math., 28:560-79, 2002.

[54] Ch. Brouder. The structure of Green functions in quantum field theory with a general state. In B. Fauser, J. Tolksdorf, and E. Zeidler, editors, Quantum Field Theory - Competitive Models, pages 163-75, Basel, 2009. Birkhäuser.

[55] R. Friedberg. Dual trees and resummation theorems. J. Math. Phys., 16:20-30, 1975.

[56] M. Livernet. A rigidity theorem for preLie algebras. J. Pure Appl. Alg., 207:1-18, 2006.

Institut de Minéralogie et de Physique des Milieux Condensés, CNRS UMR7590, Universités Paris 6 et 7, IPGP, 140 Rue de Lourmel, 75015 Paris, France.

Université de Nice, Laboratoire J.-A. Dieudonné, CNRS UmR 6621, Parc Valrose, 06108 Nice Cedex 02, France. 